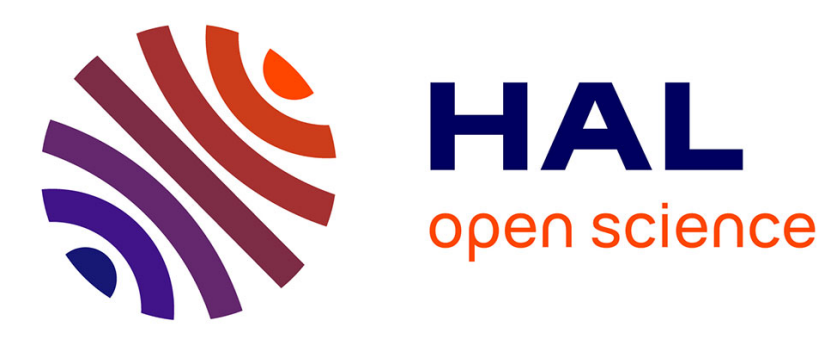

\title{
Manifold Reconstruction using Tangential Delaunay Complexes
}

Jean-Daniel Boissonnat, Arijit Ghosh

\section{To cite this version:}

Jean-Daniel Boissonnat, Arijit Ghosh. Manifold Reconstruction using Tangential Delaunay Complexes. [Research Report] RR-7142, 2009. inria-00440337v1

\section{HAL Id: inria-00440337 \\ https://hal.inria.fr/inria-00440337v1}

Submitted on 10 Dec 2009 (v1), last revised 16 Sep 2011 (v2)

HAL is a multi-disciplinary open access archive for the deposit and dissemination of scientific research documents, whether they are published or not. The documents may come from teaching and research institutions in France or abroad, or from public or private research centers.
L'archive ouverte pluridisciplinaire HAL, est destinée au dépôt et à la diffusion de documents scientifiques de niveau recherche, publiés ou non, émanant des établissements d'enseignement et de recherche français ou étrangers, des laboratoires publics ou privés. 


\section{N R I A}

INSTITUT NATIONAL DE RECHERCHE EN INFORMATIQUE ET EN AUTOMATIQUE

\section{Manifold Reconstruction using Tangential Delaunay Complexes}

Jean-Daniel Boissonnat — Arijit Ghosh

$\mathbf{N}^{\circ} 7142$

Décembre 2009

apport

de recherche 



\title{
Manifold Reconstruction using Tangential Delaunay Complexes
}

\author{
Jean-Daniel Boissonnat刚, Arijit Ghosh \\ Thème : Algorithmique, calcul certifié et cryptographie \\ Équipe-Projet Géométrica \\ Rapport de recherche $\mathrm{n}^{\circ} 7142$ - Décembre 2009 - 37 pages
}

\begin{abstract}
We give a provably correct algorithm to reconstruct a $k$-dimensional manifold embedded in $d$-dimensional Euclidean space. Input to our algorithm is a point sample coming from an unknown manifold. Our approach is based on two main ideas : the notion of tangential Delaunay complex defined in [6, 19, 20, and the technique of sliver removal by weighting the sample points [13]. Differently from previous methods, we do not construct any subdivision of the embedding $d$-dimensional space. As a result, the running time of our algorithm depends only linearly on the extrinsic dimension $d$ while it depends quadratically on the size of the input sample, and exponentially on the intrinsic dimension $k$. To the best of our knowledge, this is the first certified algorithm for manifold reconstruction whose complexity depends linearly on the ambient dimension. We also prove that for a dense enough sample the output of our algorithm is isotopic to the manifold and a close geometric approximation of the manifold.
\end{abstract}

Key-words: Tangential Delaunay complex, manifold learning, manifold reconstruction, sampling conditions, sliver exudation.

This work is partially supported by the ANR project GAIA.

* Email: Jean-Daniel.Boissonnat@sophia.inria.fr

$\dagger$ Géométrica Team, INRIA Sophia Antipolis - Méditerranée.

‡ Email: Arijit.Ghosh@sophia.inria.fr 


\section{Reconstruction de variétés avec le complexe tangent}

Résumé : Nous proposons un algorithme certifié permettant de reconstruire une variété de dimension $k$ plongée dans un espace euclidien de dimension $d$. L'entrée de l'algorithme est un ensemble fini de points échantillonnant une variété. La sortie est une approximation de cette variété. Notre approche utilise deux idées principales : la notion de complexe tangent et la technique de suppression des slivers par pondération des points de l'échantillon. Au contraire des méthodes développées auparavant, notre algorithme ne construit aucune subdivision de l'espace ambient, ce qui a pour conséquence que sa complexité ne dépend que linéairement de la dimension extrinsèque $d$; elle dépend de manière quadratique de la taille de l'échantillon et de manière exponentielle de la dimension intrinsèque $k$. A notre connaissance, c'est le premier algorithme de reconstruction dont la complexité ne dépende pas exponentiellement de la dimension extrinsèque. Nous prouvons également que si l'échantillon est suffisamment dense, la sortie de l'agorithme est une variété triangulée isotope à la variété mesurée.

Mots-clés : Complexe de Delaunay tangent, Apprentissage de variétés, Reconstruction de variétés, Conditions d'échantillonnage, Suppression des slivers 


\section{Introduction}

Manifold reconstruction consists in computing a PL approximation of an unknown manifold $\mathbb{M} \subset \mathbb{R}^{d}$ from a finite sample of unorganized points $\mathcal{P}$ lying on $\mathbb{M}$ or close to $\mathbb{M}$. When the manifold is a two-dimensional surface embedded in $\mathbb{R}^{3}$, the problem is known as the surface reconstruction problem. Surface reconstruction is a problem of major practical interest which has been extensively studied in the fields of Computational Geometry, Computer Graphics and Computer Vision. In the last decade, solid foundations have been established and the problem is now pretty well understood. Refer to Dey's book [17, and the survey by Cazals and Giesen in 9 for recent results. The output of those methods is a triangulated surface that approximates $\mathbb{M}$. This triangulated surface is usually extracted from a 3-dimensional of the ambient space (typically a grid or a triangulation). Although rather inoffensive in 3-dimensional space, such data structures depend exponentially on the dimension of the ambient space, and all attempts to extend those geometric approaches to more general manifolds has led to algorithms whose complexities depend exponentially on $d$ [27, 11, 14.

The problem in higher dimensions is also of great practical interest in data analysis and machine learning. In those fields, the general assumption is that, even if the data are represented as points in a very high dimensional space $\mathbb{R}^{d}$, they in fact live on a manifold of much smaller intrinsic dimension 29. If the manifold is linear, well-known global techniques like principal component analysis (PCA) or multi-dimensional scaling (MDS) can be efficiently applied. When the manifold is highly nonlinear, several more local techniques have attracted much attention in visual perception and many other areas of science. Among the prominent algorithms are Isomap [30, LLE [28, Laplacian eigenmaps [3, Hessian eigenmaps [18, diffusion maps [24, 26], principal manifolds [31. Most of those methods reduces to computing an eigendecomposition of some connection matrix. In all cases, the output is a mapping of the original data points into $\mathbb{R}^{k}$ where $k$ is the estimated intrinsic dimension of $\mathbb{M}$. Those methods come with guarantees only in very restricted cases (if any). For example, Isomap provides a correct embedding only if $\mathbb{M}$ is isometric to a convex open set of $\mathbb{R}^{k}$. To be able to better approximate the sampled manifold, another route is to extend the work on surface reconstruction and to construct a PL approximation of $\mathbb{M}$ from the sample in such a way that, under appropriate sampling conditions, the quality of the approximation can be guaranteed. First investigations along this line can be found in the work of Cheng, Dey and Ramos [14, and Boissonnat, Guibas and Oudot [7]. In both cases, however, the complexity of the algorithms is exponential in the ambient dimension $d$, which highly reduces their practical relevance.

In this paper, we extend the geometric techniques developped in small dimensions and propose a way to avoid computing data structures in the ambient space. We assume that $\mathbb{M}$ is a smooth manifold of known dimension $k$ and that we can compute the tangent space to $\mathbb{M}$ at any sample point. Under those conditions, we propose a provably correct algorithm that allows to construct a simplicial complex of dimension $k$ that approximates $\mathbb{M}$. The complexity of the algorithm is linear in $d$, quadratic in the size $n$ of the sample, and exponential in $k$. Our work builds on [14 and 7 but dramatically reduces the dependance on $d$, which is exponential in the cited works. To the best of our knowledge, this is the first certified algorithm for manifold reconstruction whose complexity 
depends only linearly on the ambient dimension. In the same spirit, Chazal and Oudot [12] have devised an algorithm of intrinsic complexity to solve the easier problem of computing the homology of a manifold from a sample.

Our approach is based on two main ideas : the notion of tangential Delaunay complex defined in [20, 6, 19, and the technique of sliver removal by weighting the sample points 13. The tangential complex is obtained by gluing local (Delaunay) triangulations around each sample point. The tangential complex is a subcomplex of the $d$-dimensional Delaunay triangulation of the sample points but it can be computed using mostly operations in the $k$-dimensional tangent spaces at the sample points. Hence the dependence on $k$ rather than $d$ in the complexity. However, due to the presence of so-called inconsistencies, the local triangulations may not form a triangulated manifold. Although this problem has been reported [20], no solution was known except for the case of curves $(k=1)$ [19]. We show that we can remove inconsistencies by weighting the sample points under appropriate sample conditions. We can then prove that the approximation returned by our algorithm is isotopic to $\mathbb{M}$, and a close geometric approximation of $\mathbb{M}$.

Our algorithm can be seen as a local version of the cocone algorithm of Cheng et al. 14. By local, we mean that we do not compute any $d$-dimensional data structure like a grid or a triangulation of the ambient space. Still, the tangential complex is a subcomplex of the $d$-dimensional Delaunay triangulation of the data points and therefore implicitly relies on a global partition of the ambient space. This is key to our analysis and makes our method depart from other local algorithms that have been proposed in the surface reconstruction literature [16, 22. We can also forsee applications of the tangential complex and of our construction each time computations in the tangent space of a manifold are required, e.g. for dimensionality reduction and approximating the Laplace Beltrami operator [4].

Notations. In the rest of the paper, we assume that $\mathbb{M}$ is a smooth manifold of dimension $k$ embedded in $\mathbb{R}^{d}$. We call $\mathcal{P}=\left\{p_{1}, \ldots, p_{n}\right\}$ a finite sample of points from $\mathbb{M}$. We denote by $T_{p}$ the $k$-dimensional tangent space at point $p \in \mathbb{M}$.

We write $B(c, r)$ for the $d$-dimensional ball centered at $c$ of radius $r$.

We define the angle between two vector spaces $U$ and $V$ as

$$
\angle U V=\max _{u \in U} \min _{v \in V} \angle u v .
$$

The simplex $\tau=\operatorname{conv}\left(p_{1}, \ldots, p_{i}\right)$ is also denoted by $\left[p_{1}, \ldots, p_{i}\right]$ and we identify $\tau$ with the set of its vertices when there is no ambiguity. Hence, we write $p_{j} \in \tau$ if $p_{j}$ is a vertex of $\tau$. The affine hull of a $\tau$ is denoted by aff $(\tau)$, and $\tau$ is a $j$-simplex if $j$ is the dimension of $\operatorname{aff}(\tau)$. The normal space of aff $(\tau)$ is denoted by $N_{\tau}$.

Radius and center of the smallest sphere encircling a simplex $\tau$ is denoted by $R_{\tau}$ and $c_{\tau}$ respectively . 


\section{Definitions and preliminaries}

\subsection{Weighted Delaunay triangulation}

Weighted points. A weighted point is a pair consisting of a point $p$ of $\mathbb{R}^{d}$, called the center of the weighted point, and a non-negative real number $\omega(p)$, called the weight of the weighted point. It might be convenient to visualize the weighted point $(p, \omega(p))$ as the spher ${ }^{1}$ centered at $p$ of radius $\omega(p)$.

Two weighted points (or spheres) $(p, \omega(p))$ and $(q, \omega(q))$ are called orthogonal when $\|p-q\|^{2}=\omega(p)^{2}+\omega(q)^{2}$, further than orthogonal when $\|p-q\|^{2}>$ $\omega(p)^{2}+\omega(q)^{2}$, and closer than orthogonal when $\|p-q\|^{2}<\omega(p)^{2}+\omega(q)^{2}$.

Given a point set $\mathcal{P}=\left\{p_{1}, \ldots, p_{n}\right\} \subseteq \mathbb{R}^{d}$, a weight function on $\mathcal{P}$ is a non-negative real-valued function $\omega: \mathcal{P} \rightarrow[0, \infty)$. Write $p_{i}^{\omega}=\left(p_{i}, \omega\left(p_{i}\right)\right)$ and $\mathcal{P}^{\omega}=\left\{p_{1}^{\omega}, \ldots, p_{n}^{\omega}\right\}$.

We define the relative amplitude of $\omega$, denoted as $\tilde{\omega}$, as $\max _{p \in \mathcal{P}, q \in \mathcal{P} \backslash\{p\}} \frac{\omega(p)}{\|p-q\|}$. In the paper, we assume that $\tilde{\omega} \leq \omega_{0}<1 / 2$, for some constant $\omega_{0}$ to be fixed later.

Given a subset $\tau$ of $d+1$ weighted points whose centers are affinely independent, there exists a unique sphere orthogonal to the weighted points of $\tau$. The sphere is called the orthosphere of $\tau$ and its center and radius are called the orthocenter and the orthoradius of $\tau$. If $\tau$ is a $j$-sliver, $j<d$, the orthosphere of $\tau$ is the minimal sphere that is orthogonal to the (weighted) vertices of $\tau$. Plainly, its center $o_{\tau}$ lies in aff $(\tau)$. Radius of the orthosphere of $\tau$ is denoted by $R_{\tau}^{\prime}$.

A finite set of weighted points $\mathcal{P}^{\omega}$ is said to be in general position if there exists no sphere orthogonal to $d+2$ weighted points of $\mathcal{P}^{\omega}$.

Weighted Voronoi diagram and Delaunay triangulation. Let $\omega$ be a weight function defined over $\mathcal{P}$. We define the weighted Voronoi cell of $p \in \mathcal{P}$ as

$$
\operatorname{Vor}^{\omega}(p)=\left\{x \in \mathbb{R}^{d}:\|p-x\|^{2}-\omega^{2}(p) \leq\|q-x\|^{2}-\omega^{2}(q), \forall q \in \mathcal{P}\right\} .
$$

The weighted Voronoi cells and their $k$-dimensional faces, $0 \leq k \leq d$, form a cell complex, called the weighted Voronoi diagram of $\mathcal{P}$, that decomposes $\mathbb{R}^{d}$ into convex polyhedral cells.

Let $\tau$ be a subset of points of $\mathcal{P}$ and write $\operatorname{Vor}^{\omega}(\tau)=\cap_{x \in \tau} \operatorname{Vor}^{\omega}(x)$. If the points of $\mathcal{P}$ are in general position, $\operatorname{Vor}^{\omega}(\tau)=\emptyset$ when $|\tau|>d+1$. The collection of all simplices $\operatorname{conv}(\tau)$ such that $\operatorname{Vor}^{\omega}(\tau) \neq \emptyset$ constitutes the weighted Delaunay triangulation $\operatorname{Del}^{\omega}(\mathcal{P})$. The mapping that associates to the face $\operatorname{Vor}^{\omega}(\tau)$ of $\operatorname{Vor}^{\omega}(\mathcal{P})$ the face $\operatorname{conv}(\tau)$ of $\operatorname{Del}^{\omega}(\mathcal{P})$ is a duality, i.e. a bijection that reverses the inclusion relation. For simplicity, we will use in the sequel the same notation $\tau$ to denote the simplex $\operatorname{conv}(\tau)$ and the set of its vertices.

Alternatively, a $d$-simplex $\tau$ is in $\operatorname{Del}^{\omega}(\mathcal{P})$ if the orthosphere of $\tau$ is further than orthogonal from all weighted points in $\mathcal{P}^{\omega} \backslash\left\{\tau^{\omega}\right\}$.

The weighted Delaunay triangulation of a set of weighted points can be computed efficiently in small dimensions and has found many applications, see e.g. 14, 7. In this paper, we use weighted Delaunay triangulations for two main reasons. The first one is that the restriction of a $d$-dimensional weighted

\footnotetext{
${ }^{1} \mathrm{~A}$ hypersphere is simply called a sphere when there is no ambiguity.
} 
Voronoi diagram to an affine space of dimension $k$ is a $k$-dimensional weighted Voronoi diagram that can be computed without computing the $d$-dimensional diagram (see Lemma 1). The other main reason is that some flat simplices named slivers can be removed from a Delaunay triangulation by weighting the vertices (see [14, 7, 13] and Subsections 2.3 and 4.1).

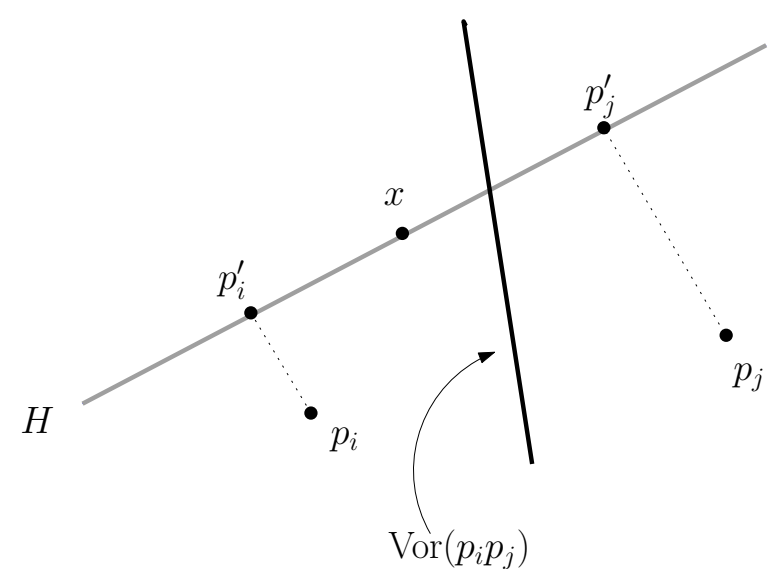

Figure 1: Refer to Lemma 1. The red line denotes the $k$-dimensional plane $H$ and the black line denotes $\operatorname{Vor}^{\omega}\left(p_{i} p_{j}\right)$.

Lemma 1 Let $H$ be a k-dimensional affine space of $\mathbb{R}^{d}$. The restriction of the weighted Voronoi diagram of $\mathcal{P}$ to $H$ is the $k$-dimensional weighted Voronoi diagram of $\mathcal{P}^{\prime}$ where $\mathcal{P}^{\prime}$ is the orthogonal projection of $\mathcal{P}$ onto $H$ and the squared weight of $p_{i}^{\prime}$ is $\omega^{2}\left(p_{i}\right)-\left\|p_{i}-p_{i}^{\prime}\right\|^{2}$.

Proof. By Pythagoras theorem, we have $\forall x \in H \cap \operatorname{Vor}^{\omega}\left(p_{i}\right), \quad\left\|x-p_{i}\right\|^{2}-$ $\omega^{2}\left(p_{i}\right) \leq\left\|x-p_{j}\right\|^{2}-\omega^{2}\left(p_{j}\right) \Leftrightarrow\left\|x-p_{i}^{\prime}\right\|^{2}+\left\|p_{i}-p_{i}^{\prime}\right\|^{2}-\omega^{2}\left(p_{i}\right) \leq\left\|x-p_{j}^{\prime}\right\|^{2}+$ $\left\|p_{j}-p_{j}^{\prime}\right\|^{2}-\omega^{2}\left(p_{j}\right)$, where $p_{i}^{\prime}$ denotes the orthogonal projection of $p_{i} \in \mathcal{P}$ onto $H$. Hence the restriction of $\operatorname{Vor}^{\omega}(\mathcal{P})$ to $H$ is the weighted Voronoi diagram of the weighted points $\left(p_{i}^{\prime}, \omega_{i}\right) \in H$ where $\omega_{i}^{2}=-\left\|p_{i}-p_{i}^{\prime}\right\|^{2}+\omega^{2}\left(p_{i}\right)$.

\subsection{Sampling conditions}

Local feature size. The medial axis of $\mathbb{M}$ is the closure of the sets of points of $\mathbb{R}^{d}$ that have more than one nearest neighbor on $\mathbb{M}$. The local feature size of $x \in \mathbb{M}$, lfs $(x)$, is the distance of $x$ to the medial axis of $\mathbb{M}$. As is well known and can be easily proved, lfs is Lipschitz continuous i.e, $\operatorname{lfs}(x) \leq \operatorname{lfs}(y)+\|x-y\|$.

$(\varepsilon, \delta)$-sample. The point sample $\mathcal{P}$ is said to be a $(\varepsilon, \delta)$-sample (where $0<$ $\delta<\varepsilon<1$ ) if (1) for any point $x \in \mathbb{M}$ there exist a point $p \in \mathcal{P}$ such that $\|x-p\| \leq \varepsilon \operatorname{lfs}(x)$, and (2) for any two distinct points $p, q \in \mathcal{P},\|p-q\| \geq \delta \operatorname{lfs}(p) .^{2}$ The ratio $\varepsilon / \delta$ is called the sparsity ratio of $\mathcal{P}$.

We will use the following results from [21]. We write $l_{p}$ for the distance between $p \in \mathcal{P}$ and its nearest neighbor in $\mathcal{P} \backslash\{p\}$.

\footnotetext{
${ }^{2}$ Observe that the sparsity condition (2) is mandatory if one wants to infer the dimension of $\mathbb{M}$ from a sample 21].
} 
Lemma $2((\varepsilon, \delta)$-SAMPLING PROPERTIES) Given an $(\varepsilon, \delta)$-sample $\mathcal{P}$ of $\mathbb{M}$, we have

1. $\delta \operatorname{lfs}(p) \leq l_{p} \leq \frac{2 \varepsilon}{1-\varepsilon} \operatorname{lfs}(p)$.

2. For any two points $p, q \in \mathbb{M}$ such that $\|p-q\|=t \operatorname{lfs}(p), 0<t<1$, $\sin \angle\left(p q, T_{p}\right) \leq t / 2$.

3. Let $p$ be a point in $\mathbb{M}$. Let $x$ be a point in $T_{p}$ such that $\|p-x\| \leq t \operatorname{lfs}(p)$ for some $0<t \leq 1 / 4$. Let $x^{\prime}$ be the point on $\mathbb{M}$ closest to $x$. Then $\left\|x-x^{\prime}\right\| \leq 2 t^{2} \operatorname{lfs}(p)$.

\subsection{Slivers and good simplices}

Consider a $j$-simplex $\tau$, where $1 \leq j \leq k$. We denote by $R_{\tau}, L_{\tau}, V_{\tau}$ and $\rho(\tau)=$ $R_{\tau} / L_{\tau}$ the circumradius, the shortest edge length, the volume, and the radiusedge ratio of $\tau$ respectively. The property of a simplex to have a good radius edge ratio is measured in terms of parameter $\rho_{0}$, i.e a simplex $\tau$ has a good radius-edge ratio implies $\rho(\tau) \geq \rho_{0}$. We define $\sigma(\tau)=V_{\tau} / L_{\tau}^{j}$, as the quality measure of $\tau$. The orthocenter of $\tau$ is denoted by $o_{\tau}$ and its orthoradius by $R_{\tau}^{\prime}$.

If $p \in \tau$, we define $\tau_{p}=\tau \backslash\{p\}$ to be the $(j-1)$-face of $\tau$ opposite to $p$. Assuming there is no ambiguity on $\tau$, we also write $D_{p}$ for the distance from $p$ to the affine hull of $\tau_{p}$, and $H_{p}$ for the distance from $o_{\tau}$ to aff $\left(\tau_{p}\right)$.

Lemma 3 ([13]) Let $\tau$ be a simplex of $\operatorname{Del}^{\omega}(\mathcal{P})$. Let $p$ be any vertex of $\tau$ and write $H(\omega(p))$ (instead of $H_{p}$ ) for the signed distance of the orthocenter of $\tau$ to $\operatorname{aff}\left(\tau_{p}\right)$ parametrized by the weight of $p$. We have $H(\omega(p))=H(0)-\frac{\omega^{2}(p)}{2 D_{p}}$.

Slivers are a special type of flat simplices. The property of being a sliver is measured in terms of a parameter $\sigma_{0}$, called the sliverity bound, to be fixed later in Section 4

Slivers are defined by induction on the dimension as in [25]: (1) a simplex of dimension less than 3 is not a sliver, and (2) for $j \geq 3$, a $j$-simplex $\tau$ is a $j$-sliver if none of its boundary simplices is a sliver and $\sigma(\tau)<\sigma_{0}^{j}$. We have the following result for $j$-slivers.

Lemma 4 If $\tau$ is a $j$-sliver then $D_{p}<j \sigma_{0} L_{\tau}$ for all vertices $p \in \tau$.

Proof. The volume of $\tau$ is $V_{\tau}=V_{\tau_{p}} . D_{p} / j=\sigma\left(\tau_{p}\right) L_{\tau_{p}}^{j-1} . D_{p} / j$ and it is also equal to $\sigma(\tau) L_{\tau}^{j}$. Since $\tau$ is a $j$-sliver we have $\sigma(\tau)<\sigma_{0}^{j}$ and $\sigma\left(\tau_{p}\right) \geq \sigma_{0}^{j-1}$. Therefore we get

$$
D_{p}=j \frac{\sigma(\tau)}{\sigma\left(\tau_{p}\right)} \times \frac{L_{\tau}^{j}}{L_{\tau_{p}}^{j-1}}<j \sigma_{0} L_{\tau}
$$

A simplex $\tau$ is called a good simplex if the radius-edge ratio of $\tau \geq \rho_{0}$ and $\tau$ nor its subsimplices are slivers.

Lemma 5 (Normal APPRoximation) Let $\tau$ be a good $j$-simplex for $j \leq k$ with vertices on a $k$-dimensional smooth manifold $\mathbb{M}$, and $p \in \tau$ s.t. the lengths of the edges of $\tau$ that are incident to $p$ are less than $b \varepsilon l \mathrm{ls}(p)$ for $b \varepsilon<1 / 4$. Then, for any normal vector $n_{p}$ of $\mathbb{M}$ at $p, \tau$ has a normal $n_{\tau}$ such that $\angle n_{p} n_{\tau} \leq a_{j} \varepsilon$, where $a_{j}$ depends on $j, \sigma_{0}, \rho_{0}$ and $b$. 
Proof. The proof is a variant of a proof of 14. The proof is by induction on $j$. For $j=0$, the claim is trivial and $a_{0}=0$. Let $\tau$ be a $j$-simplex, $p$ and $q$ two vertices of $\tau$. We write $\tau_{q}$ for the $(j-1)$-simplex $\tau \backslash\{q\}, n_{p}$ for a normal vector to $\mathbb{M}$ at $p$, and $q^{\prime}$ for the orthogonal projection of $q$ onto aff $\left(\tau_{q}\right)$.

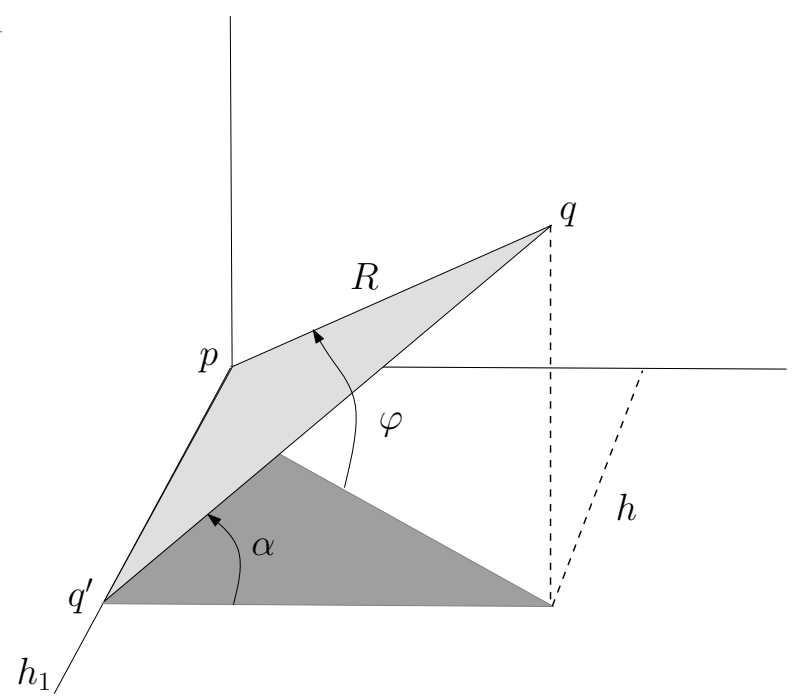

Figure 2: For the proof of Lemma 5.

We assume (induction hypothesis) that $\tau_{q}$ has a normal $n_{\tau_{q}}$ s.t $\sin \angle\left(n_{\tau_{q}}, n_{p}\right) \leq$ $a_{j-1} \varepsilon$. Let $h$ be the $(d-1)$-hyperplane containing $\tau_{q}$ and normal to $n_{\tau_{q}}$ (Refer to Fig. 7). We denote by $h_{1}$ the $(d-2)$-hyperplane of $h$ orthogonal to $q q^{\prime}$. Assume that $q \notin h$. Moreover, $h_{1}$ contains $\tau_{q}$ since $\tau_{q}$ is contained in $h$ and is normal to $q q^{\prime}$. Consider now the hyperplane $h_{2}$ that contains $h_{1}$ and $q$. Observe that $h_{2}$ contains $\tau$ and write $n_{\tau}$ for its normal.

We want to bound $\sin \angle\left(n_{\tau}, n_{p}\right) \leq \sin \angle\left(n_{\tau_{q}}, n_{p}\right)+\sin \angle\left(n_{\tau_{q}}, n_{\tau}\right)$. The first term is bounded by the induction hypothesis. Let us bound the second one. Writing $\alpha=\angle\left(n_{\tau_{q}}, n_{\tau}\right)$ and $\phi$ the angle between $p q$ and $h$ (see Fig. 7), we have

$\sin \alpha=\frac{\|p-q\|}{\left\|q-q^{\prime}\right\|} \sin \phi=\frac{\|p-q\|}{D_{q}} \sin \phi \leq \frac{2 R_{\tau}}{D_{q}} \sin \phi \leq \frac{2 \rho_{0} L_{\tau}}{D_{q}} \sin \phi \leq \frac{2^{j}}{j ! \rho_{0}^{j-2} \sigma_{0}^{j}} \sin \phi$,

as $D_{p} \geq \frac{j ! \rho(\tau)^{j-1} \sigma(\tau)}{2^{j-1}}$ by Lemma $30, L_{\tau}, \rho(\tau) \geq \rho_{0}$ and $\sigma(\tau) \geq \sigma_{0}^{j}$.

Since $\phi \leq \angle\left(n_{\tau_{q}}, n_{p}\right)+\angle\left(T_{p}, p q\right)$, we have using the induction hypothesis and Lemma 2 (2)

$$
\sin \phi \leq \sin \angle\left(n_{\tau_{q}}, n_{p}\right)+\sin \angle\left(T_{p}, p q\right) \leq\left(a_{j-1}+\frac{b}{2}\right) \varepsilon .
$$

We conclude that

$$
\sin \angle\left(n_{\tau}, n_{p}\right) \leq \frac{2^{j}}{j ! \rho_{0}^{j-2} \sigma_{0}^{j}}\left(a_{j-1}+\frac{b}{2}\right) \varepsilon+a_{j-1} \varepsilon \stackrel{\text { def }}{=} a_{j} \varepsilon .
$$




\subsection{Tangential Delaunay complex and inconsistent config- urations}

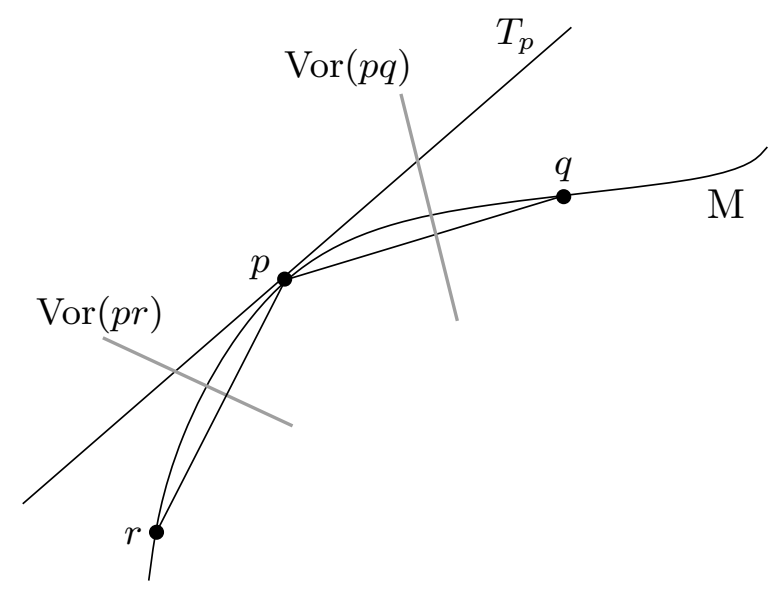

Figure 3: $[p q]$ and $[p r]$ are edges of the star of $p$ in $\operatorname{Del}_{T \mathbb{M}}^{\omega}(\mathcal{P})$ since their dual Voronoi edges intersect the tangent space $T_{p}$ at $p$.

Let $\operatorname{Del}_{p_{i}}^{\omega}(\mathcal{P})$ be the weighted Delaunay triangulation of $\mathcal{P}$ restricted to the tangent space $T_{p_{i}}$. Equivalently, the simplices of $\operatorname{Del}_{p_{i}}^{\omega}(\mathcal{P})$ are the simplices of $\operatorname{Del}^{\omega}(\mathcal{P})$ whose Voronoi dual faces intersect $T_{p_{i}}$, i.e. $\tau \in \operatorname{Del}_{p_{i}}^{\omega}(\mathcal{P})$ iff $\operatorname{Vor}^{\omega}(\tau) \cap T_{p_{i}} \neq \emptyset$. Observe that $\operatorname{Del}_{p_{i}}^{\omega}(\mathcal{P})$ is in general a $k$-dimensional triangulation. Since this situation can always be ensured by applying some infinitesimal perturbation on $\mathcal{P}$, we will assume, in the rest of the paper, that the points of $\mathcal{P}$ are in general position, meaning that all $\operatorname{Del}_{p_{i}}^{\omega}(\mathcal{P})$ are $k$-dimensional triangulations ${ }^{3}$ Finally, write $\operatorname{star}\left(p_{i}\right)$ for the star of $p_{i}$ in $\operatorname{Del}_{p_{i}}^{\omega}(\mathcal{P})$, i.e. the set of simplices that are incident to $p_{i}$ in $\operatorname{Del}_{p_{i}}^{\omega}(\mathcal{P})$.

We call tangential Delaunay complex or tangential complex for short, the simplicial complex $\{\tau, \tau \in \operatorname{star}(p), p \in \mathcal{P}\}$. We denoted it by $\operatorname{Del}_{T \mathbb{M}}^{\omega}(\mathcal{P})$. By our general position assumption, $\operatorname{Del}_{T \mathbb{M}}^{\omega}(\mathcal{P})$ is a $k$-dimensional complex contained in $\operatorname{Del}^{\omega}(\mathcal{P})$.

By duality, computing $\operatorname{star}\left(p_{i}\right)$ is equivalent to computing the restriction of the (weighted) Voronoi cell of $p_{i}$ to $T_{p_{i}}$, which, by Lemma 1, reduces to computing a cell in a $k$-dimensional weighted Voronoi diagram embedded in $T_{p_{i}}$. It follows that the tangential complex can be computed without constructing any data structure of dimension higher than $k$, the intrinsic dimension of $\mathbb{M}$.

The tangential Delaunay complex is not in general a triangulated manifold and therefore not a good approximation of $\mathbb{M}$. This is due to the presence of so-called inconsistencies. Consider a $k$-simplex $\tau$ of $\operatorname{Del}_{T \mathbb{M}}^{\omega}(\mathcal{P})$ with two vertices $p_{i}$ and $p_{j}$ such that $\tau$ is in $\operatorname{star}\left(p_{i}\right)$ but not in $\operatorname{star}\left(p_{j}\right)$. We write $B_{i}(\tau)$ for the open ball centered on $T_{p_{i}}$ that is orthogonal to the (weighted) vertices of $\tau^{\omega}$, and denote by $c_{p_{i}}$ and $r_{p_{i}}$ its center and its radius. According to our definition, $\tau$ is inconsistent iff $B_{i}(\tau)$ is further than orthogonal from all weighted points in $\mathcal{P}^{\omega} \backslash \tau^{\omega}$ while there exists a weighted point of $\mathcal{P}^{\omega} \backslash \tau^{\omega}$, say $p_{l}^{\omega}$, that is closer

\footnotetext{
${ }^{3}$ We will take care of the general position assumption in Section 7.2
} 
than orthogonal from $B_{j}(\tau)$. We deduce from the above discussion that the line segment $\left[c_{i} c_{j}\right]$ has to penetrate the interior of $\operatorname{Vor}^{\omega}\left(p_{l}\right)$.

We formally define an inconsistent configuration as follows.

Definition 1 (InCOnsistent COnfiguration) $\phi=\left[p_{1}, \ldots, p_{k+2}\right]$ is called an inconsistent configuration of $\operatorname{Del}_{T \mathbb{M}}^{\omega}(\mathcal{P})$ witnessed by $p_{i}, p_{j}, p_{l}$ if

- The $k$-simplex $\tau=\phi \backslash\left\{p_{l}\right\}$ is in $\operatorname{star}\left(p_{i}\right)$ but not in $\operatorname{star}\left(p_{j}\right)$.

- $\tau$ is a good simplex.

- $\operatorname{Vor}^{\omega}\left(p_{l}\right)$ is the first cell of $\operatorname{Vor}^{\omega}(\mathcal{P})$ whose interior is intersected by $\left[c_{i} c_{j}\right]$, where $c_{i}=T_{p_{i}} \cap \operatorname{Vor}^{\omega}(\tau)$ and $c_{j}=T_{p_{j}} \cap \operatorname{aff}\left(\operatorname{Vor}^{\omega}(\tau)\right)$, and $\left[c_{i} c_{j}\right]$ is oriented from $c_{i}$ to $c_{j}$.

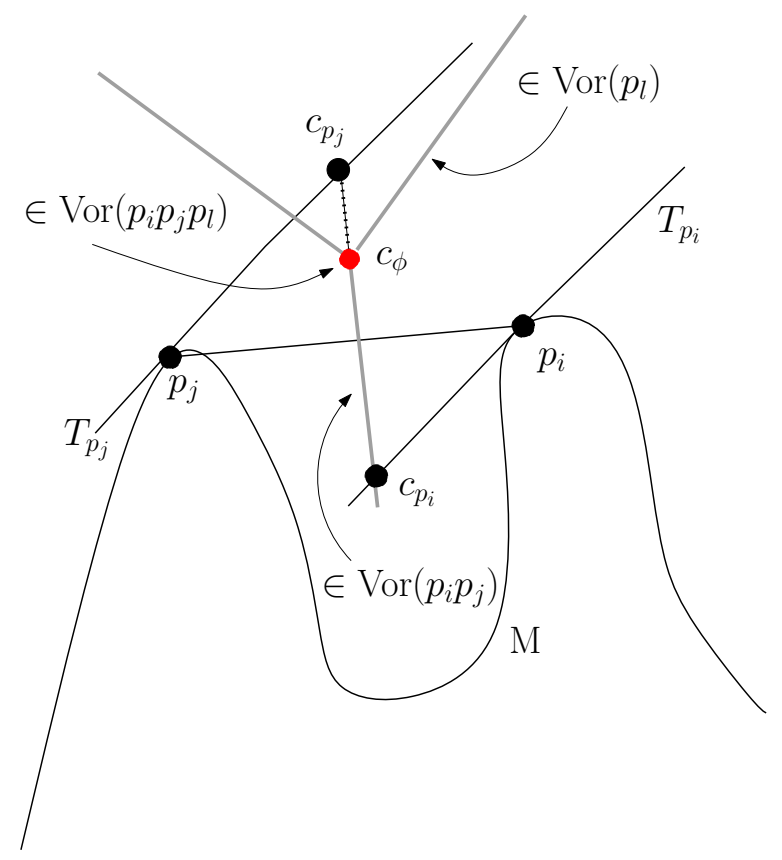

Figure 4: An inconsistent configuration in the unweighted case. Edge $\left[p_{i} p_{j}\right]$ is in $\operatorname{Del}_{p_{i}}(\mathcal{P})$ but not in $\operatorname{Del}_{p_{j}}(\mathcal{P})$ since $\operatorname{Vor}\left(p_{i} p_{j}\right)$ intersects $T_{p_{i}}$ but not $T_{p_{j}}$. This happens because $\left[c_{p_{i}} c_{p_{j}}\right]$ penetrates (at $c_{\phi}$ ) the Voronoi cell of a point $p_{l} \neq p_{i}, p_{j}$, therefore creating an inconsistent configuration $\phi=\left[p_{i}, p_{j}, p_{l}\right]$.

For an inconsistent configuration $\phi$, we denote by $c_{\phi}$ an itersection point as defined in the definition. Note that $c_{\phi}$ is the center of a sphere that is orthogonal to the weighted vertices of $\tau$ and also to $p_{l}$, and further than orthogonal from all the other weighted points of $\mathcal{P}^{\omega}$. Equivalenty, $c_{\phi}$ is the point on $\left[c_{p_{i}} c_{p_{j}}\right]$ that belongs to $\operatorname{Vor}^{\omega}(\phi)$. We call $c_{\phi}$ a witness center of $\phi$.

Hence, an inconsistent configuration is a $(k+1)$-simplex of $\operatorname{Del}^{\omega}(\mathcal{P})$. However, the subfaces of an inconsistent configuration may not belong to the tangential complex. We write Faces $^{\omega}(\mathcal{P})$ for the set of all $k$-subfaces of the inconsistent configurations of $\operatorname{Del}_{T \mathbb{M}}^{\omega}(\mathcal{P})$. 


\section{Structural results}

In the rest of the paper, we will make the following hypothesis

Hypothesis $1 \mathcal{P}$ is an $(\varepsilon, \delta)$-sample of $\mathbb{M}$ where $\varepsilon<0.09$ and whose sparsity ratio $\varepsilon / \delta$ is at most some positive constant $c_{0}$. We assume further that $\tilde{\omega} \leq \omega_{0}$ where $\tilde{\omega}$ is the relative amplitude of the weight assignement $\omega$ and $\omega_{0}$ is a positive constant less than $1 / 2$.

\subsection{Properties of the simplices of the tangential Delaunay complex}

We state now two lemmas which are slight variants of results of [14. The proofs are given in Appendix C.

Lemma 6 For all $x \in T_{p} \cap \operatorname{Vor}^{\omega}(p),\|p-x\| \leq c_{1} \varepsilon \operatorname{lfs}(p)$ if $c_{1}>2$ and $\varepsilon<$ $\frac{1}{2 c_{1}}\left(-c_{1}-1+\sqrt{c_{1}^{2}+6 c_{1}-7}\right)$. In particular we can take $c_{1}=4.41$ and $\varepsilon<0.09$.

Lemma 7 Let $c_{2}=c_{1}\left(1+1 / \sqrt{1-4 \omega_{0}^{2}}\right)$ (note that $c_{2}>2 c_{1}$ ). For $\varepsilon$ sufficiently small

1. If $p q$ is an edge of $\operatorname{Del}_{p}^{\omega}(\mathcal{P})$, then $\|p-q\| \leq c_{2} \varepsilon \operatorname{lfs}(p)$.

2. Assume that $3 c_{2} \varepsilon<1$. If $p q$ is an edge of $\operatorname{Del}_{T \mathbb{M}}^{\omega}(\mathcal{P})$, then $\|p-q\| \leq$ $3 c_{2} \varepsilon \operatorname{lfs}(p)$.

3. Let $\tau$ be a simplex in $\operatorname{Del}_{T \mathbb{M}}^{\omega}(\mathcal{P})$ and $R_{\tau}^{\prime}$ be the orthoradius of $\tau$. Then $R_{\tau}^{\prime} \leq \beta_{0} L_{\tau}$ and radius-edge ratio of $\tau$ is $\beta_{0}^{\prime}$, where $\beta_{0}=\frac{3 c_{1} \varepsilon}{2 \delta}$ and $\beta_{0}^{\prime}=$ $\frac{\beta_{0}}{\sqrt{1-4 \omega_{0}^{2}}}$.

\subsection{Properties of inconsistent configurations}

Refer to Appendix C for the proofs of the lemmas. We only give below the proof of Lemma 8 which can serve as an illustrative example.

Lemma 8 Let $c_{4}=\frac{c_{1}}{\sqrt{1-4 \omega_{0}^{2}}}, c_{5}=\frac{2 c_{4}}{\sqrt{1-4 \omega_{0}^{2}}}$ and assume that $\min \left\{12 c_{2}, 2 a_{k}\right\} \varepsilon<$

1. Let $\phi=\left[p_{1}, \ldots, p_{k+2}\right]$ be an inconsistent configuration witnessed by $p_{i}, p_{j}$, and $p_{l}$. Then 1. $\operatorname{dist}\left(c_{\phi}, \tau\right) \leq 2 a_{k} c_{4} \varepsilon^{2} \operatorname{lfs}\left(p_{i}\right)$, 2. $\left\|p_{i}-c_{\phi}\right\| \leq 2 c_{4} \varepsilon \mathrm{lfs}\left(p_{i}\right)$, and 3. $\left\|x-c_{\phi}\right\| \leq c_{5} \varepsilon \mathrm{lfs}\left(p_{i}\right)$ for all vertices $x$ of $\phi$.

Proof. From the definition of inconsistent configurations, $\tau=\phi \backslash\left\{p_{l}\right\}$ belongs to $\operatorname{Del}_{p_{i}}^{\omega}(\mathcal{P})$. By Lemma 6 . $\left\|p_{i}-c_{p_{i}}\right\| \leq c_{1} \varepsilon \mathrm{lfs}\left(p_{i}\right)$ and, by Lemma 5 . there exists a constant $a_{k}$ such that $\angle\left(\operatorname{aff}(\tau), T_{p_{i}}\right) \leq a_{k} \varepsilon$, which implies that $\tan ^{2} \angle\left(\operatorname{aff}(\tau), T_{p_{i}}\right) \leq \frac{a_{k}^{2} \varepsilon^{2}}{1-a_{k}^{2} \varepsilon^{2}}<4 a_{k}^{2} \varepsilon^{2}$. Observing that $\left\|p_{i}-o_{\tau}\right\| \leq\left\|p_{i}-c_{p_{i}}\right\|$ since $o_{\tau}$ is the closest point to $p_{i}$ in $\operatorname{aff}\left(\operatorname{Vor}^{\omega}(\tau)\right)$, we then deduce

$$
\left\|c_{p_{i}}-o_{\tau}\right\| \leq\left\|c_{p_{i}}-p_{i}\right\| \sin \angle\left(\operatorname{aff}(\tau), T_{p_{i}}\right) \leq a_{k} c_{1} \varepsilon^{2} \operatorname{lfs}\left(p_{i}\right) .
$$

We bound now $\left\|p_{j}-c_{p_{i}}\right\|$. From Lemma 31 (Appendix $(\mathrm{B})$, we have

$$
\left\|p_{j}-c_{p_{i}}\right\| \leq \frac{\left\|p_{i}-c_{p_{i}}\right\|}{\sqrt{1-4 \omega_{0}^{2}}} \leq \frac{c_{1} \varepsilon \operatorname{lfs}\left(p_{i}\right)}{\sqrt{1-4 \omega_{0}^{2}}}
$$

$\mathrm{RR} \mathrm{n}^{\circ} 7142$ 
Moreover, $\sin \angle\left(\operatorname{aff}(\tau), T_{p_{j}}\right) \leq a_{k} \varepsilon$ (Lemma 5), and $\left\|p_{j}-o_{\tau}\right\| \leq\left\|p_{j}-c_{p_{i}}\right\|$ as $o_{\tau}$ is the closest point to $p_{j}$ in $\operatorname{aff}\left(\operatorname{Vor}^{\omega}(\tau)\right)$. Hence we have,

$$
\left\|c_{p_{j}}-o_{\tau}\right\| \leq\left\|p_{j}-o_{\tau}\right\| \tan \angle\left(\operatorname{aff}(\tau), T_{p_{j}}\right)<\frac{2 a_{k} c_{1} \varepsilon^{2}}{\sqrt{1-4 \omega_{0}^{2}}} \operatorname{lfs}\left(p_{i}\right)
$$

As $c_{\phi} \in\left[c_{p_{i}} c_{p_{j}}\right]$, we conclude that $\left\|o_{\tau}-c_{\phi}\right\| \leq \frac{2 a_{k} c_{1} \varepsilon^{2}}{\sqrt{1-4 \omega_{0}^{2}}} \operatorname{lfs}\left(p_{i}\right)=2 a_{k} c_{4} \varepsilon^{2} \operatorname{lfs}\left(p_{i}\right)$. Therefore

$$
\begin{aligned}
\left\|p_{i}-c_{\phi}\right\| & \leq\left\|p_{i}-o_{\tau}\right\|+\left\|o_{\tau}-c_{\phi}\right\| \\
& \leq\left\|p_{i}-c_{p_{i}}\right\|+\left\|o_{\tau}-c_{\phi}\right\| \quad\left(\text { as }\left\|p_{i}-o_{\tau}\right\| \leq\left\|p_{i}-c_{\phi}\right\|\right) \\
& \leq c_{1} \varepsilon \operatorname{lfs}\left(p_{i}\right)+2 a_{k} c_{4} \varepsilon^{2} \operatorname{lfs}\left(p_{i}\right) \leq 2 c_{4} \varepsilon \operatorname{lfs}\left(p_{i}\right) \quad\left(\text { as } 2 a_{k} \varepsilon<1 \text { and } c_{4} \geq c_{1}\right)
\end{aligned}
$$

From Lemma 31 , we have $\left\|x-c_{\phi}\right\| \leq \frac{\left\|p_{i}-c_{\phi}\right\|}{\sqrt{1-4 \omega_{0}^{2}}}$ for all vertices $x$ of $\phi$.

The following lemma is the analogous of Lemma 7 for simplices of $\operatorname{Faces}^{\omega}(\mathcal{P})$ (which are not necessarily simplices of $\operatorname{Del}_{T \mathbb{M}}^{\omega}(\mathcal{P})$ ).

Lemma 9 Assume that $\max \left\{2 a_{k}, 16 c_{5}\right\} \varepsilon \leq 1$. Let $\tau$ be a $j$-dimensional simplex of $\operatorname{Faces}^{\omega}(\mathcal{P})$ incident to $p$.

1. If $p q$ is an edge of $\operatorname{Faces}^{\omega}(\mathcal{P})$, then $\|p-q\| \leq 4 c_{5} \varepsilon \operatorname{lfs}(p)$.

2. Let $R_{\tau}^{\prime}$ denotes the radius of the smallest orthosphere of $\tau$. Then $R_{\tau}^{\prime} \leq$ $\beta_{1} L_{\tau}$ and the radius-edge ratio of $\tau$ is $\beta_{1}^{\prime}$, where $\beta_{1}=\left(\frac{5 c_{4} \varepsilon}{2 \delta}\right)$ and $\beta_{1}^{\prime}=$ $\frac{\beta_{1}}{\sqrt{1-4 \omega_{0}^{2}}}$.

Lemma 10 Assume $\max \left\{2 a_{k}, 16 c_{5}\right\} \varepsilon<1$. Let $\tau$ be a $j$-simplex in $\operatorname{Del}_{T \mathbb{M}}^{\omega}(\mathcal{P}) \cup$ Faces $^{\omega}(\mathcal{P})$. For all vertices $x$ of $\tau$, the distance between the orthocenter of $\tau$ and $\operatorname{aff}\left(\tau_{x}\right)$ is at most $c_{6} \varepsilon \mathrm{lfs}(x)$, where $c_{6}=c_{5}\left(10+8 \beta_{1}+8 \omega_{0}\right)$.

The following lemma is analogous to Lemma 10. Instead of simplices in $\operatorname{Del}_{T \mathbb{M}}^{\omega}(\mathcal{P}) \cup \operatorname{Faces}^{\omega}(\mathcal{P})$, we consider inconsistent configurations with good faces.

Lemma 11 Assume that $\max \left\{16 c_{5}, 2 a_{k}\right\} \varepsilon<1$. Let $\phi=\left[p_{1}, \ldots, p_{k+2}\right]$ be an inconsistent configuration s.t. all $k$-subfaces are good simplices. Then the distance between $c_{\phi}$ of $\phi$ to the affine hull of any $k$-subface of $\phi$ is less than $4\left(c_{1}+c_{2}+c_{4}\right) a_{k} \varepsilon^{2} \operatorname{lfs}(v)$ for any vertex $v$ of $\phi$.

The following lemma is the analogous of Lemma 4 for inconsistent configurations, and generalizes Lemma 8 . It shows that an inconsistent configuration $\phi$ has a small $D_{p}$ for all vertices $p$ of $\phi$ and therefore behave like slivers. Moreover the inconsistent configurations lie close to $\mathbb{M}$.

Lemma 12 Assume that $\max \left\{16 c_{5}, 2 a_{k}\right\} \varepsilon<1$. Let $\phi=\left[p_{1}, \ldots, p_{k+2}\right]$ be an inconsistent configuration whose $k$-subfaces are good simplices. Then the distance $D_{p_{i}}$ between $p_{i}$ and the $k$-subface $\phi_{p_{i}}$ opposite to $p_{i}$ is less than $4\left(2 c_{5}+\right.$ $\left.a_{k}\right) c_{5} \varepsilon^{2} \operatorname{lfs}\left(p_{i}\right)$. 
Lemma 9 shows that, in order to construct $\operatorname{star}(p)$ and search for inconsistencies involving $p$, it is enough to consider the points of $\mathcal{P}$ that lie in ball $B_{p}=B\left(p, 4 c_{5} \varepsilon \operatorname{lfs}(p)\right)$. Since $\varepsilon$ and $\operatorname{lfs}(p)$ are not known in practice, we will consider instead the ball $B_{p}^{\prime}=B\left(p, 4 c_{5} c_{0} l_{p}\right)$ where $l_{x}=\min _{q \in \mathcal{P}, q \neq x}\|x-q\|$. It is easily seen that $l_{x}: \mathbb{M} \rightarrow \mathbb{R}$ is 1-Lipschitz and, by Lemma 2, we have $\delta \operatorname{lfs}(p) \leq l_{p} \leq \frac{2 \varepsilon}{1-\varepsilon} \operatorname{lfs}(p)$. It follows that $B_{p}^{\prime}$ contains $B_{p}$ if $\varepsilon / \delta \leq c_{0}$. We call $L N_{p}=B_{p}^{\prime} \cap \mathcal{P}$ the local neighborhood of $p$. The sparsity of $\mathcal{P}$, Corollary 1 (in Appendix A and a packing argument imply the following lemma.

Lemma 13 If $\max \left(2,32 c_{5} c_{0}, 2 a_{k}, 16 c_{5}\right) \varepsilon \leq 1$, the number of points of $L N_{p}$ is less than a cosntant $N=\left(128 c_{5} c_{0}+2\right)^{k} c_{0}^{k}=2^{O(k)}$.

\subsection{Range of weights for slivers and inconsistencies}

In the rest of this section, we assume that the weights of all the points except one, say $p$, are fixed. The following two lemmas bound the measure of the set of squared weights $\omega^{2}(p)$ that may create slivers incident to $p$ in $\operatorname{Del}_{T \mathbb{M}}^{\omega}(\mathcal{P}) \cup$ $\operatorname{Faces}^{\omega}(\mathcal{P})$ or inconsistent configurations incident to $p$.

Definition 2 (SLIVERITY RANGE) Let $\omega$ be a weight assignment of relative amplitude at most $\omega_{0}$ we keep fixed except for $\omega(p)$. The sliverity range of a simplex $\tau$ incident on a point $p \in \mathcal{P}$ is the measure of the set of all squared weights $\omega^{2}(p)$ for which $\tau$ is a sliver of $\operatorname{Del}_{T \mathbb{M}}^{\omega}(\mathcal{P})$ or $\operatorname{Faces}^{\omega}(\mathcal{P})$.

Lemma 14 (SLIVERITy RANGe) Assume that $\max \left\{2 a_{k}, 16 c_{5}\right\} \varepsilon<1$. Let $\tau$ be a $j$-sliver in $\operatorname{Del}_{T \mathbb{M}}^{\omega}(\mathcal{P}) \cup$ Faces $^{\omega}(\mathcal{P})$ incident on $p$. Then the sliverity range of $\tau$ w.r.t $p$ is at most $\Delta_{s}(j)=A_{j} \sigma_{0} l_{p}^{2}$ with $A_{j}=16 j c_{5} c_{6} c_{0}^{2}$.

Proof. Let $\omega(p)$ be the weight of $p$ and let $H(\omega(p))$ be the signed distance of the orthocenter of $\tau$ to aff $\left(\tau_{p}\right)$. From Lemma 10 we have $|H(\omega(p))| \leq c_{6} \varepsilon \operatorname{lfs}(p)$, for all $\tau \in \operatorname{Del}_{T \mathbb{M}}^{\omega}(\mathcal{P}) \cup \operatorname{Faces}^{\omega}(\mathcal{P})$. Moreover, using Lemma 3 and the fact that $\tau$ is a candidate $j$-sliver, $H(\omega(p))=H(0)-\frac{\omega^{2}(p)}{2 D_{p}} \leq H(0)-\frac{\omega^{2}(p)}{2 j \sigma_{0} L_{\tau}}$. It follows that the sliverity range of $\tau$ is at most $4 j \sigma_{0} L_{\tau} c_{4} \varepsilon \operatorname{lfs}(p)$. Using the facts that $L_{\tau} \leq 4 c_{5} \varepsilon \operatorname{lfs}(p)$ (from Lemma 7), $\operatorname{lfs}(p) \leq l_{p} / \delta$ and $\varepsilon / \delta \leq \rho_{0}$, the sliverity range is less than $16 j c_{5} c_{6} \sigma_{0} c_{0}^{2} l_{p}^{2}$.

The inconsistency range of a configuration is defined in a way similar to the inconsistency range of a sliver.

Definition 3 (INCONSISTENCY RANGE) Let $\omega$ be a weight assignment of relative amplitude at most $\omega_{0}$ we keep fixed except for $\omega\left(p_{i}\right)$. The inconsistency range of an inconsistent configuration $\phi=\left[p_{1}, \ldots, p_{k+2}\right]$ w.r.t $p_{i}$ is the measure of the set of all squared weights $\omega^{2}\left(p_{i}\right)$ for which all the $j$-dimensional subfaces of $\phi$ are not slivers, for $j=3, \ldots, k$, and $\phi$ is an inconsistent configuration of $\operatorname{Del}_{T M}^{\omega}(\mathcal{P})$.

Lemma 15 (InCONSISTENCy RANGe) Assume that $\max \left\{2 a_{k}, 16 c_{5}\right\} \varepsilon<1$. The inconsistency range of any configuration $\phi=\left[p_{1}, \ldots, p_{k+2}\right]$ is at most $\Delta_{i}=$ $B \varepsilon^{2} l_{p_{i}}^{2}$ with $B=\left(c_{1}+c_{2}+c_{4}\right)\left(2 c_{5}+a_{k}\right) a_{k} c_{5} c_{0}^{2}$.

Proof. Since $\phi$ is an inconsistent configuration, hence it implies that $\phi$ belongs to $\operatorname{Del}^{\omega}(\mathcal{P})$. Let $p_{i}$ be any vertex of $\phi$ and $H\left(\omega\left(p_{i}\right)\right)$ denotes the signed distance 
of the orthocenter of $\phi$ from $\phi_{p_{i}} . H\left(\omega\left(p_{i}\right)\right)$ is positive if $p_{i}$ and the orthocenter of $\phi$, denoted by $o$, lie on the same side. From Lemma 3 , we have

$$
H\left(\omega\left(p_{i}\right)\right)=H(0)-\frac{\omega^{2}\left(p_{i}\right)}{2 D_{p_{i}}} .
$$

Since $\phi$ is an inconsistent configuration with no faces being a slivers, we have from Lemma 11 the distance between $c_{\phi}$ and aff $\left(\phi_{p_{i}}\right)$ is at most $h=4\left(c_{1}+c_{2}+\right.$ $\left.c_{4}\right) a_{k} \varepsilon^{2} \operatorname{lfs}\left(p_{i}\right)$. And $H\left(\omega\left(p_{i}\right)\right)=\operatorname{dist}\left(o_{\phi} \text {, aff }\left(\phi_{p_{i}}\right)\right)^{4}$ is less than the distance of $c_{\phi}$ from aff $\left(\phi_{p_{i}}\right)$. With $\operatorname{lfs}\left(p_{i}\right) \leq l_{p_{i}} / \delta$ and $\varepsilon / \delta \leq c_{0}$, we get $H\left(\omega\left(p_{i}\right)\right) \leq h \leq$ $4\left(c_{1}+c_{2}+c_{4}\right) a_{k} c_{0} \varepsilon l_{p_{i}}$.

Moreover, since $\phi$ is an inconsistent configuration, hence the distance $D_{p_{i}}$ between $p_{i}$ and aff $\left(\phi_{p_{i}}\right)$ is at most $4\left(2 c_{5}+a_{k}\right) c_{5} c_{0} \varepsilon l_{p_{i}}$.

Hence, if $\omega(p)$ and $\omega^{\prime}(p)$ are two weights for which $\phi$ is an inconsistent configuration, we have $\mid H\left(\omega\left(p_{i}\right)-H\left(\omega^{\prime}\left(p_{i}\right)\left|=\frac{1}{2 D_{p_{i}}}\right| \omega^{2}\left(p_{i}\right)-\omega^{\prime 2}(p) \mid \leq 2 h\right.\right.$, from which we deduce that the inconsistency range of $\phi$ w.r.t to $p_{i}$ is at most

$$
4 h D \leq 64\left(c_{1}+c_{2}+c_{4}\right)\left(2 c_{5}+a_{k}\right) a_{k} c_{5} c_{0}^{2} \times \varepsilon^{2} l_{p_{i}}^{2}=B \varepsilon^{2} l_{p_{i}}^{2} .
$$

\section{Inconsistencies removal}

In this section, we will show how to find a weight assignment for the points of $\mathcal{P}$ so as to remove all inconsistent configurations. Once this is done, all stars become coherent and the resulting tangential complex is a simplicial $k$-manifold.

This idea has already been successfully applied to remove slivers in 3 and higher dimensions [14, 13, 7]. The basic reasons why this approach works in our context are the following. If $\operatorname{Del}_{T \mathbb{M}}^{\omega}(\mathcal{P}) \operatorname{nor} \operatorname{Faces}^{\omega}(\mathcal{P})$ contain slivers, inconsistencies in $\operatorname{Del}_{T \mathbb{M}}^{\omega}(\mathcal{P})$ can only happen as inconsistent configurations. Thanks to Lemmas 14 and 15 , the sliverity ranges of the slivers of $\operatorname{Del}_{T \mathbb{M}}^{\omega}(\mathcal{P})$ and $\operatorname{Faces}^{\omega}(\mathcal{P})$ are small, as well as the inconsistency ranges of the inconsistent configurations. It follows that we can remove any sliver in $\operatorname{Del}_{T \mathbb{M}}^{\omega}(\mathcal{P})$ or $\operatorname{Faces}^{\omega}(\mathcal{P})$, and any inconsistent configuration by weighting one of their vertices. Lastly, we observe that, thanks to Lemma 13 , there are only a constant number of simplices of dimensions at most $k+1$ that may appear in $\operatorname{Del}^{\omega}(\mathcal{P})$ and be incident to a given point of $\mathcal{P}$. Hence, we can remove all slivers of $\operatorname{Del}_{T \mathbb{M}}^{\omega}(\mathcal{P})$ and $\operatorname{Faces}^{\omega}(\mathcal{P})$ and all inconsistent configurations that are incident to a given point $p$ by weighting $p$.

As before, we assume that $k$ is given as well as an upper bound $c_{0}$ on the sparsity of $\mathcal{P}$. We choose for the bound $\omega_{0}$ on the relative amplitude $\tilde{\omega}$ of the weight assignment $\omega$ any value in the interval $[0,1 / 2)$. The constants $c_{1}, c_{2}, c_{3}$, $c_{4}$ and the number $N$ can be then computed. Assuming that all those constants have been fixed, it will remain to fix $\sigma_{0}$, which will also fix $a_{k}$ (that depends on $c_{5}, \sigma_{0}$ and $\left.\rho_{0}=\beta_{1}^{\prime}\right)$.

\subsection{Algorithm: removing slivers and inconsistencies}

The algorithm consists of computing a weight assignment $\omega$ such that no inconsistent configuration remain in $\operatorname{Del}_{T \mathbb{M}}^{\omega}(\mathcal{P})$. The overall algorithm consists of

\footnotetext{
${ }^{4} o_{\phi}$ is the orthocenter of $\phi$.
} 
two main steps. First, we compute a set of weight assignments $\omega$ so that no $j$-slivers appear in $\operatorname{Del}_{T \mathbb{M}}^{\omega}(\mathcal{P}) \cup \operatorname{Faces}^{\omega}(\mathcal{P})$ for $3 \leq j \leq k$. We proceed by increasing dimensions of the slivers to be removed and weight each point $p_{i}$ of $\mathcal{P}$ in turn. Initially all weights are set to 0 and they can only be increased during the course of the algorithm.

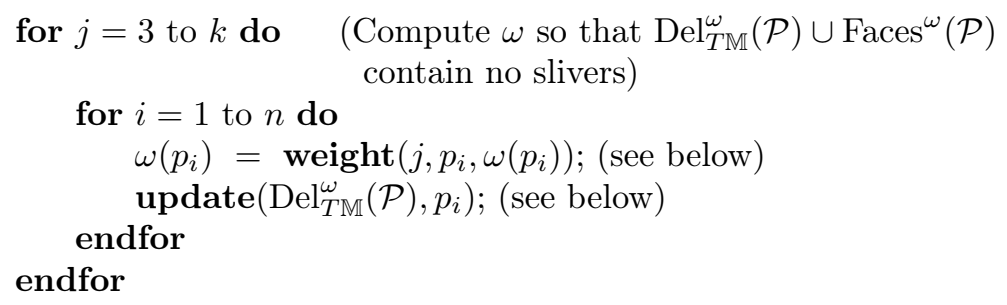

We weight each point in turn a second time so as to remove all inconsistent configurations in $\operatorname{Del}_{T \mathbb{M}}^{\omega}(\mathcal{P})$. The initial weights are the weights provided by the previous procedure. As before, weights can only increase.

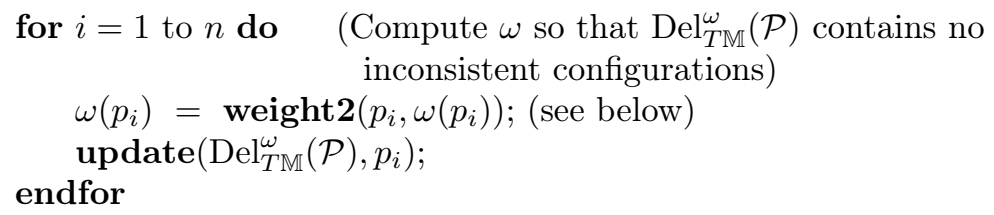

Upon termination, $\operatorname{Del}_{T \mathbb{M}}^{\omega}(\mathcal{P})$ contains no inconsistencies, i.e. any simplex appears in the stars of all its vertices.

Function update $\left(\operatorname{Del}_{T \mathbb{M}}^{\omega}(\mathcal{P}), p\right)$

- Update the stars of all points $x \in L N_{p}$ by modifying $\operatorname{Del}_{x}^{\omega}\left(L N_{x}\right)$ if $p \in$ $L N_{x}$.

\section{Function weight $(j, p, \omega(p))$}

1. Call function detect candidate-slivers $\left(j^{\prime}, p\right)$ for all $3 \leq j^{\prime} \leq j$, to find all the so-called candidate $j^{\prime}$-slivers, i.e. $j^{\prime}$-slivers incident to $p$ that appear in $\operatorname{Del}_{T \mathbb{M}}^{\omega}(\mathcal{P})$ or in $\operatorname{Faces}^{\omega}(\mathcal{P})$ when $\omega(p)$ varies (the other weights remaining fixed).

2. Select the minimum weight in the interval $\left[\omega(p), \omega_{0} l_{p}\right]$ for which $\operatorname{Del}_{T \mathbb{M}}^{\omega}(\mathcal{P})$ and Faces $^{\omega}(\mathcal{P})$ contain no $j^{\prime}$-slivers for $3 \leq j^{\prime} \leq j$ calculated in step 1 are incident on $p$.

\section{Function detect candidate-slivers $(j, p)$}

1. We first detect all possible $j$-simplices of $\operatorname{Del}_{T M}^{\omega}(\mathcal{P})$ incident on $p$ for all possible $\omega(p)$. This is done in the following way: (1) we vary the weight of $p$ from 0 to its maximum, which is $\omega_{0} l_{p}$, keeping the weights of the other points constant (2) for each new weight assignment to $p$ we modify the stars of the points in $L N_{p}{ }^{5}$ and detect from the stars the new $j$-simplices

\footnotetext{
${ }^{5}$ We don't have to calculate the stars of the points outside $L N_{p}$ as they won't contain any simplices incident to $p$.
} 
incident to $p$ that have not been detected thus far. The weight of point $p$ changes only in a finite number of instances

$$
0=P_{0}<P_{1}<\cdots<P_{n-1}<P_{n}=\omega_{0} l_{p} .
$$

We determine the next weight assignment of $p$ in the following way. For each new simplex $\tau$ currently incident to $p$, we keep it in a priority queue ordered by the weight of $p$ at which $\tau$ will be destroyed for the first time. Hence the minimum weight in the priority queue gives the next weight assignment for $p$. Since the number of points in $L N_{p}$ is bounded, the number of simplices incident to $p$ is also bounded, as well as the number of times we have to change the weight of $p$.

2. As the new $j$-simplices are generated, we detect the ones that are $j$-slivers.

3. Once we have detected all possible $j$-slivers incident on $p$, we find out all possible inconsistent configurations incident to $p$, by calling the function detect_inconsistent-configuration $(p)$. From the inconsistent configurations, we extract the $j$-slivers of $\operatorname{Faces}^{\omega}(\mathcal{P})$ that are incident to $p$.

\section{Function detect_inconsistent-configuration $(p)$}

1. We vary the weight of $p$ from 0 to $\omega_{0} l_{p}$, keeping the weight of the rest of the points constant. Once we have assigned a new weight to $p$ we modify the stars of the points in $L N_{p}$.

2. Detecting the inconsistent configurations incident to $p$ once we have modified the stars of the points in $L N_{p}$ is more complicated than detecting the simplices incident to $p$. We consider all points $p_{i}$ in $L N_{p}$. Let $\tau$ be a $k$-simplex in the star of $p_{i}$, and let $p_{j}$ be a vertex of $\tau$ such that $\tau$ is not in the star of $p_{j}$. We calculate the Voronoi diagram of the points in $L N_{p}$ restricted to the line segment $\left[c_{p_{i}} c_{p_{j}}\right]$, where $c_{p_{i}}=T_{p_{i}} \cap \operatorname{Vor}^{\omega}(\tau)$ and $c_{p_{j}}=T_{p_{j}} \cap \operatorname{aff}\left(\operatorname{Vor}^{\omega}(\tau)\right)$. From the restricted Voronoi diagram, we find a point $r$ whose Voronoi cell intersects for the first time the line segment $\left[c_{p_{i}} c_{p_{j}}\right]$ oriented from $c_{p_{i}}$ to $c_{p_{j}}$. If $p \in \phi=\tau \cup\{r\}$, then we report $\phi$.

3. As in the detect candidate-slivers function the weight of $p$ is changed only a finite number of times. For each current inconsistent configuration $\phi$ incident to $p$, we keep it in a priority queue the weight of $p$ for which $\phi$ will be destroyed for the first time. The minimum weight in the priority queue gives the next weight assignment of $p$.

\section{Function weight2 $(p, \omega(p))$}

1. Call functions detect_candidate-slivers $(j, p)$ for $3 \leq j \leq k$, and detect inconsistent-configuration $(p)$ to find all the slivers and inconsistent configurations incident on $p$.

2. Select a weight in the interval $\left[\omega(p), \omega_{0} l_{p}\right]$ for which no $j$-slivers for $3 \leq$ $j \leq k$ nor any inconsistent configurations calculated in step 1 are incident to $p$. 
Lemma 16 Function weight returns a weight for $p_{i}$ such that there is no candidate $j^{\prime}$-sliver nor any inconsistent configuration incident to $p_{i^{\prime}}$ for all $j^{\prime} \leq k$ and $i^{\prime} \leq i$.

Proof. We call new simplex a candidate simplex that appears in $\operatorname{Del}_{T \mathbb{M}}^{\omega}(\mathcal{P})$ after changing the weight of $p_{i}$. Since the weight are always increasing, a new simplex is either a $k$-simplex incident to $p_{i}$ or a subface of such a $k$-simplex.

The proof is by induction. The assertion is trivially true for $j<3$. Assume that the assertion holds for weight $\left(j^{\prime}, p_{i^{\prime}}\right)$ for all $j^{\prime}<j$ and all $i^{\prime}<i$. We now prove that after applying Function weight $\left(j, p_{i}\right)$, the assertion holds for all $j^{\prime} \leq j$ and all $i^{\prime} \leq i$. In fact, it is sufficient to prove the assertion for the new simplices by the remark above.

Function weight $\left(j, p_{i}\right)$ does not create any candidate $j^{\prime}$-sliver incident to $p_{i}$ for all $j^{\prime} \leq j$ (Step 1). It cannot create $j^{\prime \prime}$-slivers, $j^{\prime \prime}<j$, that do not contain $p_{i}$ either. Indeed, such a $j^{\prime \prime}$-sliver should have existed before applying weight $\left(j, p_{i}\right)$, which would contradict the induction hypothesis.

Lemma 17 Function weight2 returns a weight for $p_{i}$ such that there is no candidate $j^{\prime}$-sliver in $\operatorname{Del}_{T \mathbb{M}}^{\omega}(\mathcal{P})$ nor in $\operatorname{Faces}^{\omega}(\mathcal{P})$, and no inconsistent configuration incident to $p_{i^{\prime}}$ for all $j^{\prime} \leq k$ and $i^{\prime} \leq i$.

Proof. The proof that $\operatorname{Del}_{T \mathbb{M}}^{\omega}(\mathcal{P})$ and $\operatorname{Faces}^{\omega}(\mathcal{P})$ contain no candidate $j^{\prime}$ slivers, $j^{\prime} \leq k$ and $i^{\prime} \leq i$ is the same as for function weight.

Consider the case of inconsistent configurations. We first observe that, since a new inconsistent configuration must contain a new $k$-simplex, it must be incident to $p_{i}$. As weight $2\left(p_{i}\right)$ does not create any inconsistent configuration incident to $p_{i}$ (Step 2), the lemma is proved.

We conclude from Lemma 16 and 17 that the tangential Delaunay complex for the weight assignment $\omega$ output by the overall algorithm does not contain any inconsistent configuration.

Let us summarize our result in the following theorem.

Theorem 1 (TERmination CONDitions) If $\mathcal{P}$ is an $(\varepsilon, \delta)$-sample of $\mathbb{M}$ and if the following properties are satisfied,

1. $\omega_{0} \in[0,1 / 2)$;

2. $\varepsilon>\delta \geq c_{0} \varepsilon>0$ for some known $c_{0}$;

3. $\varepsilon<\varepsilon_{0}=\min \left(\frac{1}{2 c_{1}}\left(-c_{1}-1+\sqrt{c_{1}^{2}+6 c_{1}-7}\right), \frac{1}{2 a_{k}}, \frac{1}{16 c_{5}}, \frac{1}{32 c_{5} c_{0}}\right)$;

4. $A_{k} \sigma_{0}+B \varepsilon^{2} \leq \frac{\omega_{0}^{2}}{N^{k+2}}$ (Pumping equation), where $A_{k}$ and $N$ are absolute constants, and $B$ depends on $\sigma_{0} \rho_{0}=\max \left\{\beta_{0}^{\prime}, \beta_{1}^{\prime}\right\}$ and $k$;

then, the above algorithm outputs $\operatorname{Del}_{T \mathbb{M}}^{\omega}(\mathcal{P})$ without any candidate slivers or inconsistent configuration.

Proof. Hypotheses (1) - (3) are needed for the structural results proved earlier in Sections 2, 3 to hold.

We have seen that the algorithm modifies the weight of a given point $k-1$ times. Let $\omega_{2}(p)=0, \omega_{3}(p), \ldots, \omega_{k+1}(p)$ be the sequence of weights assigned to 
$p$. For all $3 \leq i \leq k, \omega_{i}(p)$ is the weight assigned to the point $p$ s.t no $j^{\prime}$-slivers are incident on the point $p$ for the $3 \leq j^{\prime} \leq i$. From the weight function, we know that for all $3 \leq i \leq k$, we have the following (1) $\omega_{i}(p) \in\left[\omega_{i-1}(p), \omega_{0} l_{p}\right]$, and $(2) \omega_{i}(p)$ is the least weight s.t no $j^{\prime}$-slivers are incident on the point $p$ for the $3 \leq j^{\prime} \leq i$.

From Lemmas 14, 13 we have

$$
\begin{aligned}
\left|\omega_{i}^{2}(p)-\omega_{i-1}^{2}(p)\right| & \leq \sum_{3 \leq j \leq i}\left(N^{j+1} A_{j} \sigma_{0} l_{p}^{2}\right) \\
& \leq\left(\sum_{3 \leq j \leq i} N^{j+1}\right) A_{i} \sigma_{0} l_{p}^{2}<2 N^{i+1} A_{i} \sigma_{0} l_{p}^{2}
\end{aligned}
$$

the last inequality follows from the fact that $\sum_{3 \leq j \leq i} N^{j+1}<2 N^{i+1}$. Therefore

$$
\begin{aligned}
\left|\omega_{k}^{2}(p)-\omega_{2}^{2}(p)\right| & =\sum_{3 \leq i \leq k}\left|\omega_{i}^{2}(p)-\omega_{i-1}^{2}(p)\right| \\
& <\sum_{3 \leq i \leq k} 2 N^{i+1} A_{i} \sigma_{0} l_{p}^{2}<4 N^{k+1} A_{k} \sigma_{0} l_{p}^{2} .
\end{aligned}
$$

From the weight2 function, we have $(1) \omega_{k+1}(p) \in\left[\omega_{k}(p), \omega_{0} l_{p}\right]$, and $(2)$ $\omega_{k+1}(p)$ is a weight s.t no $j^{\prime}$-slivers for $3 \leq j^{\prime} \leq k$ and inconsistent configurations incident on $p$.

From Lemmas 14, 15 and 13, measure of the sets of squared weights of $p$ that will create a $j$-sliver for $3 \leq j \leq k$ or an inconsistent configuration incident to $p$ is less than

$$
\gamma=\sum_{3 \leq i \leq k} N^{i+1} \times A_{i} \sigma_{0} l_{p}^{2}+N^{k+2} B \varepsilon^{2} l_{p}^{2}<2 N^{k+1} A_{k} \sigma_{0} l_{p}^{2}+N^{k+2} B \varepsilon^{2} l_{p}^{2}
$$

Combining Equations 2 and 3 we get

$$
\begin{aligned}
\left|\omega_{k}^{2}(p)-\omega_{2}^{2}(p)\right|+\gamma & <6 N^{k+1} A_{k} \sigma_{0} l_{p}^{2}+N^{k+2} B \varepsilon^{2} l_{p}^{2} \\
& \leq N^{k+2} A_{k} \sigma_{0} l_{p}^{2}+N^{k+2} B \varepsilon^{2} l_{p}^{2} . \quad(\text { as } N \geq 6)
\end{aligned}
$$

If the above quantity is less than $\omega_{0}^{2} l_{p}^{2}$, the above algorithm will terminate and find a weight assignment that removes all slivers of dimensions up to $k$ from $\operatorname{Del}_{T \mathbb{M}}^{\omega}(\mathcal{P})$ and $\operatorname{Faces}^{\omega}(\mathcal{P})$, and all inconsistent configurations from $\operatorname{Del}_{T \mathbb{M}}^{\omega}(\mathcal{P})$.

\subsection{Time complexity}

Before the total time complexity of the algorithm is calculated we first calculate the individual time complexity for each function.

At each point $p \in \mathcal{P}$ we maintain $\operatorname{Del}_{p}^{\omega}\left(L N_{p}\right)$. We need to calculate $L N_{p}$ for all points $p \in \mathcal{P}$ once. The total time complexity to do this operation is $O\left(d|\mathcal{P}|^{2}\right)$.

From Lemma 1, the time complexity of updating $\operatorname{Del}_{p}^{\omega}\left(L N_{p}\right)$ is

$$
\left(d\left|L N_{p}\right|+k^{3} O\left(\left|L N_{p}\right|^{\left\lceil\frac{k}{2}\right\rceil}\right)\right)=d 2^{O\left(k^{2}\right)} .
$$


The last equality follows from the fact that $\left|L N_{p}\right| \leq N=2^{O(k)}$ from Lemma 13 . The constant in the big- $O$ is independent of $d$ (see Lemma 13).

Function update $\left(\operatorname{Del}_{T \mathbb{M}}^{\omega}(\mathcal{P}), p\right)$ modifies $\operatorname{Del}_{p}^{\omega}\left(L N_{p}\right)$ as well as $\operatorname{Del}_{x}^{\omega}\left(L N_{x}\right)$ for all the points $x \in L N_{p}$. Since we assume that $\mathcal{P}$ is a dense sample satisfying the first three hypothesis of Theorem 11 we have $\operatorname{Del}_{p}^{\omega}(\mathcal{P})=\operatorname{Del}_{p}^{\omega}\left(L N_{p}\right)$, and $\left|L N_{p}\right| \leq N=2^{O(k)}$ for all $p \in \mathcal{P}$. Hence for calculating $\operatorname{Del}_{p}^{\omega}(\mathcal{P})$, we only need to calculate the restricted weighted Delaunay triangulation of $L N_{p}$ restricted to $T_{p}$. Since we modify $\operatorname{Del}_{x}^{\omega}\left(L N_{p}\right)$ for all the points in $L N_{p}$, we have the total time complexity of update $\left(\operatorname{Del}_{T \mathbb{M}}^{\omega}(\mathcal{P}), p\right)$ is less than

$$
d 2^{O\left(k^{2}\right)} \times\left|L N_{p}\right|=d 2^{O\left(k^{2}\right)} \times N=d 2^{O\left(k^{2}\right)} .
$$

Function detect_inconsistent-configuration $(p)$ detects all the possible inconsistent configurations incident to $p$. We vary the weight of $p$ in increasing order from 0 to $\omega_{0} l_{p}$. The number of times $\omega(p)$ is changed is equal to number of possible inconsistent-configuration incident to $p$, which is atmost $\left|L N_{p}\right|^{k+1}=$ $2^{O\left(k^{2}\right)}$. We also need to calculate for each inconsistent configuration $\phi$ the minimum weight $\omega(p)$ of $p$ for which $\phi$ will be destroyed for the first time. The time complexity of this operation is $O\left(d k^{3}\right)$, since we need to calculate the sign of determinant of $k \times k$ matrix. Once the weight of $p$ is fixed, we calculate the stars of all the points in $L N_{p}$, which takes less than $d 2^{O\left(k^{2}\right)}$ time. Then all $k$-simplices in the stars of all points in $L N_{p}$ are checked for inconsistencies. If a given simplex $\tau$ is inconsistent then the time complexity to find the inconsistent configuration $\phi$ corresponding to $\tau$ is $d L N_{p}=d 2^{O(k)}$. The total number of $k$-simplices we need to check is less $\left|L N_{p}\right| \times 2^{O\left(k^{2}\right)}=2^{O\left(k^{2}\right)}$. Hence the total time complexity of detect_inconsistent-configuration $(p)$ is less than

$$
2^{O\left(k^{2}\right)}\left(O\left(d k^{3}\right)+d 2^{O\left(k^{2}\right)}+d 2^{O(k)} \times 2^{O\left(k^{2}\right)}\right)=d 2^{O\left(k^{2}\right)} .
$$

Similarly the time complexity for detect_candidate-slivers $(j, p)$ is $d 2^{O\left(k^{2}\right)}$.

For the function weight $(j, p, \omega(p))$ we first need to calculate all the possible $j^{\prime}$-slivers that can be incident on $p$, for all $3 \leq j^{\prime} \leq j$ by calling detect_candidateslivers $\left(j^{\prime}, p\right)$. The time complexity of this step is $(j-3) \times d 2^{O\left(k^{2}\right)}=\bar{d} 2^{O\left(k^{2}\right)}$, as time complexity of detect candidate-slivers $(j, p)$ is $d 2^{O\left(k^{2}\right)}$. Then we find the minimum weight of $p$ in the range $\left[\omega(p), \omega_{0} l_{p}\right]$ that will remove all the slivers calculated in the first step. This operation takes $j^{3} 2^{O\left(k^{2}\right)}=2^{O\left(k^{2}\right)}$ time. Hence the total time complexity of weight $(j, p, \omega(p))$ is less than $d 2^{O\left(k^{2}\right)}$.

Similarly, the time complexity of the function weight $\left(p_{i}, \omega\left(p_{i}\right)\right)$ is $d 2^{O\left(k^{2}\right)}$.

In the course of the algorithm for each point $p \in \mathcal{P}$, we call weight $(j, p, \omega(p))$ once for all $3 \leq j \leq k$ and weight2 $(p, \omega(p))$ once. Adding everything up, the time compelxity for our algorithm is

$$
d|\mathcal{P}|^{2}+(k-2) \times d 2^{O\left(k^{2}\right)}|\mathcal{P}|=d|\mathcal{P}|^{2}+d 2^{O\left(k^{2}\right)}|\mathcal{P}| .
$$

\subsection{Space complexity}

Storing the point sample $\mathcal{P}$ which lies in $d$-dimensional space requires $d|\mathcal{P}|$ space.

Storing $\operatorname{Del}_{p}^{\omega}\left(L N_{p}\right)$ at each point $p$ of $\mathcal{P}$ requires

$$
\sum_{p \in \mathcal{P}} d O\left(k\left|L N_{p}\right|^{\left\lceil\frac{k}{2}\right\rceil}\right) \leq d 2^{O\left(k^{2}\right)}|\mathcal{P}|,
$$


space. The last inequality follows from the fact that $\left|L N_{p}\right| \leq N=2^{O(k)}$ for all points $p \in \mathcal{P}$ (refer to Lemma 13).

Therefore the total space complexity of the algorithm is

$$
d|\mathcal{P}|+d 2^{O\left(k^{2}\right)}|\mathcal{P}|=d 2^{O\left(k^{2}\right)}|\mathcal{P}| .
$$

\section{Topological and geometric guarantees}

In this section it is assumed that $\operatorname{Del}_{T \mathbb{M}}^{\omega}(\mathcal{P})$ has no slivers and inconsistent configurations. These assumptions are justified as the $\operatorname{Del}_{T \mathbb{M}}^{\omega}(\mathcal{P})$ returned by the algorithm given in Subsection 4.1 has these properties, refer to Theorem 1

\subsection{Local Properties}

Let $\pi: \mathbb{R}^{d} \rightarrow \mathbb{M}$ maps each point of $\mathbb{R}^{d}$ to its closest point of $\mathbb{M}$. PL manifold $\operatorname{Del}_{T \mathbb{M}}^{\omega}(\mathcal{P})$ is has the following properties if $\varepsilon$ is sufficiently small.

Lemma 18 (Properties of $\operatorname{Del}_{T \mathbb{M}}^{\omega}(\mathcal{P})$ )

1. (Continuity of $\pi$ on $\operatorname{Del}_{T \mathbb{M}}^{\omega}(\mathcal{P})$ ) The map $\pi$ restricted to $\operatorname{Del}_{T \mathbb{M}}^{\omega}(\mathcal{P})$ is well defined and continuous.

2. (PieCEWISE-LINEAR MANIfOld) $\operatorname{Del}_{T \mathbb{M}}^{\omega}(\mathcal{P})$ is a picewise-linear manifold without boundaries.

3. $\operatorname{Del}_{T \mathbb{M}}^{\omega}(\mathcal{P})$ has a vertex in each connected component of $\mathbb{M}$.

4. (Small Simplex CONDition) Every simplex $\tau$ of $\operatorname{Del}_{T M}^{\omega}(\mathcal{P})$ has a circumsphere of radius $\leq c_{2} \varepsilon \mathrm{lfs}(x)$, where $x$ is any vertex of $\tau$.

5. (Flat Simplex CONDition) Given a $k$-simplex $\tau$ and a vertex $p$ of the simplex, then $\angle N_{\tau} N_{p} \leq a_{k} \varepsilon$.

\section{Proof.}

1. Let $\tau$ be a simplex in $\operatorname{Del}_{T \mathbb{M}}^{\omega}(\mathcal{P})$ and $p$ a vertex of $\tau$. Since $\operatorname{Del}_{T \mathbb{M}}^{\omega}(\mathcal{P})$ does not contain any inconsistent configuration, hence $\tau$ belongs to $\operatorname{Del}_{p}^{\omega}(\mathcal{P})$. From Lemma 7 we know that $\tau \subseteq B\left(p, c_{2} \varepsilon \mathrm{lfs}(p)\right) \subseteq B(p, 0.5 \mathrm{lfs}(p))$. Therefore $\operatorname{Del}_{T \mathbb{M}}^{\omega}(\mathcal{P})$ does not contain any point of the medial axis of $\mathbb{M}$ and $\pi$ is continuous at all points except the medial axis of $\mathbb{M}$. Hence the restriction of $\pi$ to $\operatorname{Del}_{T \mathbb{M}}^{\omega}(\mathcal{P})$ is continuous.

2. To prove that $\operatorname{Del}_{T \mathbb{M}}^{\omega}(\mathcal{P})$ is a piecewise linear manifold without boundary we have to show that for all point $p \in \mathcal{P}, p$ does not lie on the boundary of $\operatorname{star}(p)$. Above sufficiency condition follows if we can show that $\operatorname{Vor}^{\omega}(p) \cap$ $T_{p}$ is bounded. From Lemma 6 we have $\operatorname{Vor}^{\omega}(p) \cap T_{p} \subseteq B\left(p, c_{1} \varepsilon \mathrm{lfs}(p)\right)$. Hence for all point $p \in \mathcal{P}, p$ does not lie on the boundary of $\operatorname{star}(p)$, which implies $\operatorname{Del}_{T \mathbb{M}}^{\omega}(\mathcal{P})$ is a piecewise linear manifold without boundary.

3. Every point $p \in \mathcal{P}$ is a vertex of $\operatorname{Del}_{T \mathbb{M}}^{\omega}(\mathcal{P})$ and we assume point sample $\mathcal{P}$ has one point from every connected component of $\mathbb{M}$. 
4. Let $\tau$ be a simplex of $\operatorname{Del}_{T \mathbb{M}}^{\omega}(\mathcal{P})$. Since $\operatorname{Del}_{T \mathbb{M}}^{\omega}(\mathcal{P})$ has no inconsistencies hence $\tau$ is in $\operatorname{star}(x) \subseteq \operatorname{Del}_{x}^{\omega}(\mathcal{P})$ for all vertex $x$ of $\tau$. Since $\tau$ is in $\operatorname{Del}_{x}^{\omega}(\mathcal{P})$, hence from Lemma 7 we have $\tau$ is contained in $B\left(x, c_{2} \varepsilon \operatorname{lfs}(x)\right)$.

5. Let $\tau$ be a $k$-simplex of $\operatorname{Del}_{T \mathbb{M}}^{\omega}(\mathcal{P})$. From Lemma 18 (4) we know that for any vertex $p$ of $\tau, \tau$ is contained in $B\left(p, c_{2} \varepsilon\right.$ lfs $\left.(p)\right)$. We know that sliverity constant and radius-edge ratio of $\operatorname{Del}_{T \mathbb{M}}^{\omega}(\mathcal{P})$ is $\sigma_{0}$ and $\beta_{0}$ respectively. From Lemma 5 we have $\angle N_{p} N_{\tau} \leq a_{k} \varepsilon$.

Using ideas from [2, 8, and the above properties, we show $\operatorname{Del}_{T \mathbb{M}}^{\omega}(\mathcal{P})$ is isotopic to $\mathbb{M}$.

\subsection{Isotopy}

Now we prove the final lemmas that will prove the isotopy between $\operatorname{Del}_{T \mathbb{M}}^{\omega}(\mathcal{P})$ and $\mathbb{M}$. We prove the isotopy between $\operatorname{Del}_{T \mathbb{M}}^{\omega}(\mathcal{P})$ and $\mathbb{M}$ using the map $\pi$ restricted to $\operatorname{Del}_{T \mathbb{M}}^{\omega}(\mathcal{P})$. We first show in Lemma 19 (extension of Lemma 16 in [1] to higher dimension and weighted case) that $\pi$ is injective on points of $\mathcal{P}$, i.e for all points $p \in \mathcal{P}$ and $x \in \operatorname{Del}_{T \mathbb{M}}^{\omega}(\mathcal{P}), \pi(x)=p \Longrightarrow x=p$.

Definition 4 (ADJACENT $j$-SIMPLICES) Two $j$-simplices are said to be adjacent iff they share a common $(j-1)$-dimensional face.

Then in Lemma 20 (extension of Lemma 18 in [1] to higher dimension), we show that $\pi$ restricted to adjacent simplices is also injective. In Lemma 21 we show that the restriction of $\pi$ to $\operatorname{Del}_{T \mathbb{M}}^{\omega}(\mathcal{P})$ is injective using Lemmas 19 and 20. In Lemma 22 we show that the restriction of $\pi$ to $\operatorname{Del}_{T \mathbb{M}}^{\omega}(\mathcal{P})$ is surjective using the facts that the restriction of $\pi$ to $\operatorname{Del}_{T \mathbb{M}}^{\omega}(\mathcal{P})$ is injective, $\mathbb{M}$ is a manifold without boundary and $\mathcal{P}$ contains points from each connected components of M. Finally in Theorem 2 we prove the isotopy using the previous results.

Lemma 19 Let $p \in \mathcal{P}, n_{p} \in N_{p}$ be a unit vector and $l_{p}$ the normal fiber $\left[p, p+\operatorname{lfs}(p) \cdot n_{p}\right]$, then no $k$-simplex intersects the interior of the segment $l_{p}$.

Proof. Let $N$ denote the ball centered at $m=p+\operatorname{lfs}(p) \cdot n_{p}$ and tangent to the manifold at $p$. In order to intersect segment $p m$, a $k$-simplex $s \in \operatorname{Del}_{T \mathbb{M}}^{\omega}(\mathcal{P})$ would have to intersect $N$, and so would the smallest orthogonal Delaunay ball $B$ of $s$, centered at $x$ with radius $r$. Let $H$ denote the $k-1$-flat space passing through the intersection of $N$ and $B$.

The $(d-1)$ flat space $H$ decomposes the ball $N$ into two sections $N^{+}=$ $N \cap H^{+}, N^{-}=N \cap H^{-}$and the $(k-1)$ ball $N \cap H$, where $H^{+}$and $H^{-}$are the two open half spaces formed by $H$. Similarly, $B$ is also decomposed by $H$ into $B^{+}, B^{-}$and $B \cap H$. It follows from geometry that if $N^{+} \subset B^{+}$then $B^{+}$ and $N^{-}$lie on opposite sides of $H$. Without loss of generality we can assume that $N^{+} \subset B^{+}$.

Since the vertices of $s$ lie on $\mathbb{M}$, and hence not in the interior of $N, s \subset B^{+}$. Since $s$ belongs to weighted Delaunay triangulation, hence

$$
\|p-x\| \geq \sqrt{r^{2}+\omega^{2}(p)} \geq r .
$$




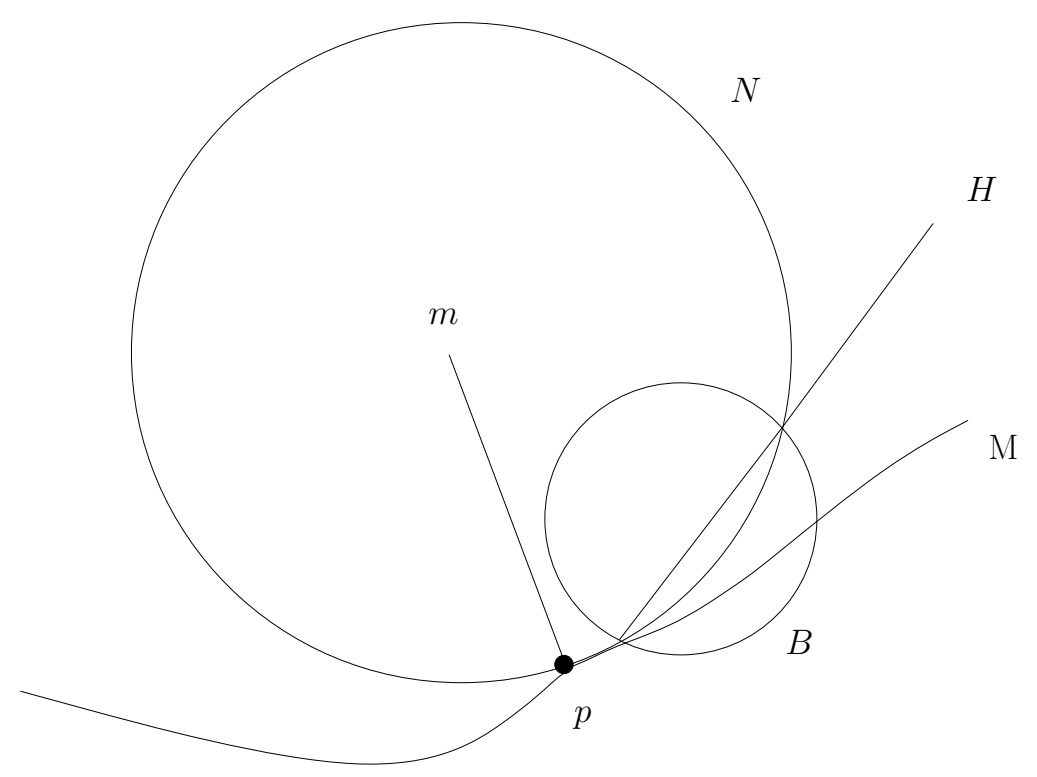

Figure 5: Refer to Lemma 19. Figure is taken from [2] and shows the case when the points are not weighted.

So $p$ cannot lie in the interior of $B$ hence $p \notin H^{+}$. We will show that $m \notin H^{+}$. If $m$ was inside $H^{+} \subset B^{+}$, the radius of $B$ would be at least $1 / 2 \operatorname{lfs}(v)$ for any vertex $v \in s$, but if we choose $\varepsilon$ small enough to make sure it is less that $1 / 2 \operatorname{lfs}(v)$, then we will reach a contradiction. Therefore $p, m$ and hence the fiber $l_{p}$ lies in $H^{-}$proving that $H$ separates $s$ and $l_{p}$.

We assume that $\varepsilon$ small enough such that $\arcsin \left(a_{k} \varepsilon\right) \leq \pi / 8$.

Lemma 20 Let $\tau_{1}$ and $\tau_{2}$ be two $k$-dimensional adjacent simplices of $\operatorname{Del}_{T \mathbb{M}}^{\omega}(\mathcal{P})$. Then the map $\pi$ restricted to $\tau_{1} \cup \tau_{2}$ is injective.

Proof. Assume, for a contradiction that there exist two points $y, z$ in $\tau_{1} \cup \tau_{2}$ such that $\pi(y)=\pi(z)=x$. Let $p$ be a common vertex of $\tau_{1}$ and $\tau_{2}$. Since we assume that $\operatorname{Del}_{T \mathbb{M}}^{\omega}(\mathcal{P})$ has no inconsistencies hence both $\tau_{1}$ and $\tau_{2}$ belong to $\operatorname{Del}_{p}^{\omega}(\mathcal{P})$. Therefore from Lemma 2(3) and Lemma 7) (1) we have

$$
\|p-x\| \leq\left(c_{2} \varepsilon+2 c_{2}^{2} \varepsilon^{2}\right) \operatorname{lfs}(p) .
$$

For $\varepsilon$ sufficiently small we get $\|p-x\| \leq 2 c_{2} \varepsilon$. Hence from Normal variation Lemma 26, we get $\angle N_{p} N_{x} \leq \arcsin \left(8 c_{2} \varepsilon\right)$. We consider the following two cases.

Case 1: W.l.o.g assume that two points lie on the same $k$-simplex $\tau_{1}$. The line segments connecting $y, x$ (denoted by $l_{y x}$ ) and $z, x$ (denoted by $l_{z x}$ ) are normals at $x$. This implies the vector $\overrightarrow{y z} \in N_{x}$ and we have reached a contradiction as $N_{x}$ and $N_{\tau}$ are almost parallel (as $\angle N_{x} N_{p} \leq \arcsin \left(8 c_{2} \varepsilon\right)$ ).

Case 2: Now assume that $y$ and $z$ lie on different simplices.

Claim 1 Two adjacent $k$-simplex in $\operatorname{Del}_{T \mathbb{M}}^{\omega}(\mathcal{P})$ meet at an angle greater than $\pi-2 \arcsin \left(a_{k} \varepsilon\right)$. 
Proof. Let $\tau_{1}$ and $\tau_{2}$ be two adjacent $k$-simplex in $\operatorname{Del}_{T \mathbb{M}}^{\omega}(\mathcal{P})$, let $\tau$ be the shared $(k-1)$-simplex shared by the two simplex, and let $p$ be a vertex of $\tau$. As $\operatorname{Del}_{T \mathbb{M}}^{\omega}(\mathcal{P})$ has no inconsistencies hence both $\tau_{1}, \tau_{2}$ belong to $\operatorname{Del}_{p}^{\omega}(\mathcal{P})$. Therefore we have two empty ortho-spheres $B_{1}, B_{2}$ for $\tau_{1}, \tau_{2}$ respectively, centered at point $v_{1}, v_{2}$ of $T_{p}$. The boundaries of the ortho-spheres $B_{1}$ and $B_{2}$ intersect in a $(d-1)$-dimensional ortho-sphere $C$ contained in a hyperplane $H$, with $\tau \subseteq H$. The line passing through $v_{1}$ and $v_{2}$ is perpendicular to $H$, hence $\angle H N_{p}=0$. Since $\omega_{0}<1 / 2$ implies $p \in \operatorname{Vor}^{\omega}(p)$, we have $v_{1}$ and $v_{2}$ lying on the opposite side of $H$. Since $\tau_{1}$ and $\tau_{2}$ are not slivers, hence $\angle N_{\tau_{i}} N_{p} \leq \arcsin \left(a_{k} \varepsilon\right)$ for $i=1$, 2, from Lemma 5. Hence smaller angle at which $\tau_{1}$ and $\tau_{2}$ meet is greater than $\pi-2 \arcsin \left(a_{k} \varepsilon\right)$.

Hence from above Claim 1. the smaller angle at which $\tau_{1}$ and $\tau_{2}$ meet is greater than $\pi-2 \arcsin \left(a_{k} \varepsilon\right)$. Since we have already shown that $y$ and $z$ cannot lie on the same simplex hence $y$ and $z$ cannot lie on the common $(k-1)$ dimensional face of both $\tau_{1}$ and $\tau_{2}$. The lines $l_{y x}$ and $l_{z x}$ are normal at the point $x$, this implies vector $\overrightarrow{y z} \in N_{x}$. This implies the vector $\overrightarrow{y z}$ intersect both $\tau_{1}, \tau_{2}$ at almost right angles but that contradicts the Claim 1 which says $\tau_{1}, \tau_{2}$ meet at an angle greater than $\pi-2 \arcsin \left(a_{k} \varepsilon\right) \geq 3 \pi / 4$.

Lemma 21 (Injectivity Lemma) For $\varepsilon \leq \varepsilon_{0}$, the map $\pi: \operatorname{Del}_{T \mathbb{M}}^{\omega}(\mathcal{P}) \rightarrow \mathbb{M}$ is injective.

Proof. We will first show that $\left(\operatorname{Del}_{T M \mathbb{M}}^{\omega}(\mathcal{P}), \pi\right)$ is a covering space of $\pi\left(\operatorname{Del}_{T \mathbb{M}}^{\omega}(\mathcal{P})\right)$. Lets denote $\operatorname{Del}_{T \mathbb{M}}^{\omega}(\mathcal{P}), \pi\left(\operatorname{Del}_{T \mathbb{M}}^{\omega}(\mathcal{P})\right)$ by $N, N^{\prime}$ respectively. The $(N, \pi)$ is a covering space of $N^{\prime}$ if, for every $x \in N^{\prime}$, there is a pathconnected elementary neighborhood $V_{x}$ around $x$ such that each path-connected component of $\pi^{-1}\left(V_{x}\right)$ is mapped homeomorphically onto $V_{x}$ by $\pi$.

To construct $V_{x}$, we note that the set of points $\left|\pi^{-1}(x)\right|$ corresponding to a point $x \in N^{\prime}$ is non-zero and finite, since $\pi$ is injective on each triangle of $N$ and there are only a finite a triangles. For each point $q \in \pi^{-1}(x)$ take an open neighborhood $U_{q}$ of around $q$, homeomorphic to a disk and small enough so that $U_{q}$ is contained only in $k$-simplex that contain $q$. We claim that $\pi$ maps each $U_{q}$ homeomorphically onto $\pi\left(U_{q}\right)$. This is because it is continuous, it is onto $\pi\left(U_{q}\right)$ by definition, and, since any two points $x$ and $y$ in $U_{q}$ are in adjacent triangles, it is one-to-one by Lemma 20. Let $U^{\prime}(x)=\cap_{q \in \pi^{-1}(x)} \pi\left(U_{q}\right)$, the intersection of the maps of each of the $U_{q}$. $U^{\prime}(x)$ is the intersection of a finite number of open neighborhood, each containing $x$, so we can find an open disk $V_{x}$ around $x$. $V_{x}$ is path connected, and each component of $\pi^{-1}\left(V_{x}\right)$ is a subset of some $U_{q}$ and hence is mapped homeomorphically onto $V_{x}$ by $\pi$. Therefore it follows that $(N, \pi)$ is a covering space for $N^{\prime}$.

We can show that $\pi$ defines a homeomorphism between $N$ and $N^{\prime}$. Since $N$ is onto $N^{\prime}$ by definition, we need only show that $\pi$ is one-to-one. Consider one connected component $C$ of $N^{\prime}$. From properties covering space [10, 23], we know that the sets $\pi^{-1}(x)$ for all $x \in G$ have the same cardinality. We know from lemma 19 that $\pi$ is one-to-one at every sample point. Since each connected component of $M$ contains some samples, hence it follows directly the $\pi$ is one-to-one, and $N$ and $N^{\prime}$ are homeomorphic.

Lemma 22 (SURJeCTIVITY LeMma) Let $\mathcal{P}$ be a $T$-loose $\varepsilon$-sample and $\varepsilon \leq \varepsilon_{0}$. The map $\pi: \operatorname{Del}_{T \mathbb{M}}^{\omega}(\mathcal{P}) \rightarrow \mathbb{M}$ is surjective. 
Proof. This follows directly from the fact that $\pi$ is injective, and $\operatorname{Del}_{T \mathbb{M}}^{\omega}(\mathcal{P})$ has no boundaries hence it cannot contain a part of the connected component of $\mathbb{M}$, and hence $\pi\left(\operatorname{Del}_{T \mathbb{M}}^{\omega}(\mathcal{P})\right)$ must consist of a subset of a connected components of $\mathbb{M}$. Since $\mathcal{P}$ contains at least one point from every connected component hence every connected components will be covered by the map $\pi$.

Theorem 2 (Isotopy Lemma) For $\varepsilon<\varepsilon_{0}$, $\operatorname{Del}_{T \mathbb{M}}^{\omega}(\mathcal{P})$ and $\mathbb{M}$ are isotopic.

Proof. The fact that $\mathbb{M}$ is homeomorphic to $\operatorname{Del}_{T \mathbb{M}}^{\omega}(\mathcal{P})$ follows directly from Lemmas 21 and 22. The map $\pi$ restricted to $\operatorname{Del}_{T \mathbb{M}}^{\omega}(\mathcal{P})$ provides the isotopy to M.

\subsection{Hausdorff Distance}

In this section we bound the distance from well-formed $\operatorname{Del}_{T \mathbb{M}}^{\omega}(\mathcal{P})$ to $\mathbb{M}$, and then use the surjectivity of $\pi$ to show that the bound also holds for the distance from $\mathbb{M}$ to $\operatorname{Del}_{T \mathbb{M}}^{\omega}(\mathcal{P})$. M.

With Lemmas 2 and 18 , we can bound the distance between $\operatorname{Del}_{T \mathbb{M}}^{\omega}(\mathcal{P})$ to

Lemma 23 Every point $q \in \operatorname{Del}_{T \mathbb{M}}^{\omega}(\mathcal{P})$ is at most $2.5\left(c_{2} \varepsilon\right)^{2} \operatorname{lfs}(p) \leq 2.5\left(c_{2} \varepsilon\right)^{2} \times$ $\max _{x \in \mathcal{P}}\{\operatorname{lfs}(x)\}$ from $\mathbb{M}$, where $p$ is a vertex of the simplex $\tau$ containing $q$ in $\operatorname{Del}_{T \mathbb{M}}^{\omega}(\mathcal{P})$, and $\varepsilon \leq \varepsilon_{0}$.

Proof. Since $\operatorname{Del}_{T \mathbb{M}}^{\omega}(\mathcal{P})$ has no inconsistent configuration therefore $\tau$ belongs to $\operatorname{star}(p)$ in $\operatorname{Del}_{p}^{\omega}(\mathcal{P})$. For all vertex $x$ of $\tau$ we have from Lemmas 7 , $\|p-x\| \leq$ $c_{2} \varepsilon$ lfs $(p)$. From Lemma 2 we have $\sin \angle\left(p x, T_{p}\right) \leq c_{2} \varepsilon / 2$. Therefore the distance between $x$ and $T_{p}$, denoted by $\operatorname{dist}\left(x, T_{p}\right)$,

$$
\operatorname{dist}\left(x, T_{p}\right)=\|p-x\| \sin \angle\left(p x, T_{p}\right) \leq\left(c_{2} \varepsilon\right)^{2} / 2 . \operatorname{lfs}(p) .
$$

Since $q$ is a point in the simplex $\tau$, we have from above calculation, the distance between $q$ and $T_{p}$ is less than $\left(c_{2} \varepsilon\right)^{2} / 2$. lfs $(p)$.

Let $q^{\prime}$ be the point on $T_{p}$ closest $q$. Since $\|p-q\| \leq c_{2} \varepsilon \operatorname{lfs}(p)$, so $\left\|p-q^{\prime}\right\| \leq$ $c_{2} \varepsilon \operatorname{lfs}(p)$. Let $q^{\prime \prime}$ be the point closest to $q^{\prime}$ on $\mathbb{M}$. From Lemma 2, we have $\left\|q^{\prime}-q^{\prime \prime}\right\| \leq 2\left(c_{2} \varepsilon\right)^{2} \operatorname{lfs}(p)$.

So from triangle inequality we get

$\left\|q-q^{\prime \prime}\right\| \leq\left\|q-q^{\prime}\right\|+\left\|q^{\prime}-q^{\prime \prime}\right\| \leq 2.5\left(c_{2} \varepsilon\right)^{2} \operatorname{lfs}(p) \leq 2.5\left(c_{2} \varepsilon\right)^{2} . \max _{x \in \mathcal{P}}\{\operatorname{lfs}(x)\}$,

as $\left\|q-q^{\prime}\right\|=\operatorname{dist}\left(q, T_{p}\right) \leq 0.5\left(c_{2} \varepsilon\right)^{2} \operatorname{lfs}(p)$ and $\left\|q^{\prime}-q^{\prime \prime}\right\| \leq 2\left(c_{2} \varepsilon\right)^{2} \operatorname{lfs}(p)$. Hence distance between $x$ and $\operatorname{Del}_{T \mathbb{M}}^{\omega}(\mathcal{P})$, denoted by $\operatorname{dist}\left(x, \operatorname{Del}_{T \mathbb{M}}^{\omega}(\mathcal{P})\right)$,

$$
\operatorname{dist}(q, \mathbb{M}) \leq\left\|q-q^{\prime \prime}\right\| \leq 2.5\left(c_{2} \varepsilon\right)^{2} \operatorname{lfs}(p) \leq 2.5\left(c_{2} \varepsilon\right)^{2} . \max _{x \in \mathcal{P}}\{\operatorname{lfs}(x)\} .
$$

Lemma 24 Every point $x \in \mathbb{M}$ is at distance at most $3\left(c_{2} \varepsilon\right)^{2} \operatorname{lfs}(x) \leq 3\left(c_{2} \varepsilon\right)^{2} \times$ $\max _{y \in \mathbb{M}}\{\operatorname{lfs}(y)\}$ from $\operatorname{Del}_{T \mathbb{M}}^{\omega}(\mathcal{P})$ and $\varepsilon \leq \varepsilon_{0}$. 
Proof. Let $x \in \mathbb{M}$. Since the restriction of $\pi$ to $\operatorname{Del}_{T \mathbb{M}}^{\omega}(\mathcal{P})$ is surjective, we have $x^{\prime} \in \operatorname{Del}_{T \mathbb{M}}^{\omega}(\mathcal{P})$ such that $\pi\left(x^{\prime}\right)=x$. According to Theorem 23. $\| x^{\prime}-$ $x \| \leq 2.5 \varepsilon^{2} \operatorname{lfs}(p) \leq 2.5 \varepsilon^{2}$. $\max _{x \in \mathcal{P}} \operatorname{lfs}(x)$, where $p$ is any vertex of the simplex containing $x^{\prime}$ in $\operatorname{Del}_{T \mathbb{M}}^{\omega}(\mathcal{P})$.

Therefore we have

$$
\begin{aligned}
\|x-p\| & \leq\left\|x^{\prime}-p\right\|+\left\|x-x^{\prime}\right\| \\
& \leq\left(c_{2} \varepsilon+2.5\left(c_{2} \varepsilon\right)^{2}\right) \operatorname{lfs}(p) \\
& \leq\left(c_{2} \varepsilon+2.5\left(c_{2} \varepsilon\right)^{2}\right)(\operatorname{lfs}(x)+\|x-p\|) \quad \text { (as lfs has 1-Lipschitz property). }
\end{aligned}
$$

Arranging the inequality we get

$$
\|x-p\| \leq \frac{c_{2} \varepsilon+2.5\left(c_{2} \varepsilon\right)^{2}}{1-c_{2} \varepsilon-2.5\left(c_{2} \varepsilon\right)^{2}} \operatorname{lfs}(x) .
$$

We can choose the $\varepsilon \leq \varepsilon_{0}$ (a constant) such that $\|x-p\| \leq 0.2 \operatorname{lfs}(x)$. Hence,

$$
\left\|x-x^{\prime}\right\| \leq 2.5\left(c_{2} \varepsilon\right)^{2} \operatorname{lfs}(p) \leq 2.5\left(c_{2} \varepsilon\right)^{2}(\operatorname{lfS}(x)+\|x-p\|) \leq 3\left(c_{2} \varepsilon\right)^{2} \operatorname{lfs}(x) .
$$

Hence distance between $x$ and $\operatorname{Del}_{T \mathbb{M}}^{\omega}(\mathcal{P})$, denoted by $\operatorname{dist}\left(x, \operatorname{Del}_{T \mathbb{M}}^{\omega}(\mathcal{P})\right)$, is

$$
\operatorname{dist}\left(x, \operatorname{Del}_{T \mathbb{M}}^{\omega}(\mathcal{P})\right) \leq\left\|x-x^{\prime}\right\| \leq 3\left(c_{2} \varepsilon\right)^{2} \operatorname{lfs}(x) \leq 3\left(c_{2} \varepsilon\right)^{2} . \max _{y \in \mathbb{M}} \operatorname{lfs}(y) .
$$

From Lemmas 23 and 24, we get the following bound on the Hausdroff distance between $\operatorname{Del}_{T \mathbb{M}}^{\omega}(\mathcal{P})$ and $\mathbb{M}$ :

Theorem 3 (HAUSDORFF DISTANCE) Let $\mathbb{M}$ be a manifold without boundary and $\operatorname{Del}_{T \mathbb{M}}^{\omega}(\mathcal{P})$ be well-formed, with $\varepsilon \leq \varepsilon_{0}$. Then the Hausdorff distance between $\mathbb{M}$ and $\operatorname{Del}_{T \mathbb{M}}^{\omega}(\mathcal{P})$ is at most $3\left(c_{2} \varepsilon\right)^{2} . \max _{x \in \mathbb{M}} \operatorname{lfs}(x)$.

\section{Summary of results}

Combining Theorem 1 and results from Section 5 we get the following theorem.

Theorem 4 (FINAL THEOREM) If $\mathcal{P}$ is an $(\varepsilon, \delta)$-sample of $\mathbb{M}$ and if the following conditions are satisfied,

- $\omega_{0} \in[0,1 / 2)$;

- $\varepsilon>\delta \geq c_{0} \varepsilon>0$ for some known $c_{0}$;

- $\varepsilon<\varepsilon_{0}=\min \left(\frac{1}{2 c_{1}}\left(-c_{1}-1+\sqrt{c_{1}^{2}+6 c_{1}-7}\right), \frac{1}{2 a_{k}}, \frac{1}{16 c_{5}}, \frac{1}{32 c_{5} c_{0}}\right)$;

- $A_{k} \sigma_{0}+B \varepsilon^{2} \leq \frac{\omega_{0}^{2}}{N^{k+2}}$ (Pumping equation), where $A_{k}$ and $N$ are absolute constants, and $B$ depends on $\sigma_{0}, \rho_{0}=\max \left\{\beta_{0}^{\prime}, \beta_{1}^{\prime}\right\}$ and $k$;

- $\arcsin \left(a_{k} \varepsilon\right) \leq \pi / 8$.

then, the algorithm outputs $\operatorname{Del}_{T \mathbb{M}}^{\omega}(\mathcal{P})$ without any sliver nor inconsistent configuration, and $\operatorname{Del}_{T \mathbb{M}}^{\omega}(\mathcal{P})$ has the following properties 
- Bijection

The projection $\pi: \operatorname{Del}_{T \mathbb{M}}^{\omega}(\mathcal{P}) \rightarrow \mathbb{M}$ is a bijection;

- Pointwise approximation

For all $x \in \mathbb{M}$, $\operatorname{dist}\left(x, \pi^{-1}(x)\right)=O\left(\varepsilon^{2} \operatorname{lfs}(x)\right)$;

- Normal approximation

For all $x \in \mathbb{M}, \angle N_{x} N_{\tau}=O(\varepsilon)$, where $\tau$ is a k-simplex of $\operatorname{Del}_{T \mathbb{M}}^{\omega}(\mathcal{P})$ containing the point $\pi^{-1}(x)$;

- Topological correctness

$\operatorname{Del}_{T \mathbb{M}}^{\omega}(\mathcal{P})$ is isotopic to $\mathbb{M}$.

\section{$7 \quad$ Extensions}

\subsection{Dimension and tangent space estimation.}

The reconstruction algorithm given here works if the dimension of $\mathbb{M}$ is known as well as the tangent space at each point of $\mathcal{P}$. Algorithms are known to compute the dimension of a manifold from a finite sample. Some of them can be used to estimate tangent spaces [15, 21]. Our algorithm is robust to small perturbation of the tangent spaces.

\subsection{Removing the general position assumption}

It is assumed that the points are in general position. This section will show how this assumption can be removed. The idea behind the calculations done here is similar to one done in Section 8 of [14].

The following properties of $\operatorname{Del}_{T \mathbb{M}}^{\omega}(\mathcal{P})$ will be used

1. The radius-edge ratio of any simplex in $\operatorname{Del}_{T \mathbb{M}}^{\omega}(\mathcal{P})$ is more than equal to $\beta_{0}^{\prime}$, refer to Lemma 6

2. Let $\tau$ be a $k$-dimensional simplex in $\operatorname{Del}_{T \mathbb{M}}^{\omega}(\mathcal{P})$ such that it is not a sliver. Then $\angle\left(T_{p}\right.$, aff $\left.(\tau)\right) \leq a_{k} \varepsilon \forall p \in \tau$, refer to Lemmas 6 and 5 .

Lemma 25 Let $k \geq 2, \tau$ be a $(k+1)$-dimensional $\operatorname{Del}_{T \mathbb{M}}^{\omega}(\mathcal{P})$ and

$$
\varepsilon<\frac{\sigma_{0}(k+1)}{\beta_{0}^{\prime}\left(3 c_{2}+2 a_{k}\right)} .
$$

If the subfaces of $\tau$ are not slivers, then $\tau$ is a sliver.

Proof. Let $p q$ be the smallest edge of the simplex $\tau$ and let $r$ be a vertex in $\tau \backslash\{p, q\}$. Since $\tau_{r}$ is not a sliver (as all subfaces of $\tau$ are not slivers), we have as $\sin \angle\left(\operatorname{aff}\left(\tau_{r}\right), T_{x}\right) \leq a_{k} \varepsilon$ for all vertices $x$ of $\tau_{r}$, refer Lemma 5 . We have from Lemma $7\|p-r\| \leq 3 c_{2} \mathrm{lfs}(p)$, and $\sin \angle\left(p r, T_{p}\right) \leq 3 c_{2} \varepsilon / 2$ from Lemma 2 . Therefore

$$
\begin{aligned}
D_{p} & =\sin \angle\left(p r, \operatorname{aff}\left(\tau_{r}\right)\right)\|p-r\| \\
& \leq \sin \left(\angle\left(p r, T_{p}\right)+\angle\left(\operatorname{aff}\left(\tau_{r}\right), T_{p}\right)\right)\|p-r\| \\
& \leq\left(\sin \angle\left(p r, T_{p}\right)+\sin \angle\left(\operatorname{aff}\left(\tau_{r}\right), T_{p}\right)\right)\|p-r\| \\
& \leq\left(3 c_{2} / 2+a_{k}\right) \varepsilon\|p-r\| .
\end{aligned}
$$


Hence

$$
\begin{aligned}
\operatorname{vol}(\tau) & =D_{r} \times \frac{\operatorname{vol}\left(\tau_{r}\right)}{(k+1)} \\
& \leq\left(a_{k}+3 c_{2} / 2\right) \varepsilon\|p-r\| \times \frac{\sigma\left(\tau_{p}\right) L_{\tau}^{k}}{(k+1)}\left(\operatorname{as~} \operatorname{vol}\left(\tau_{r}\right)=\sigma\left(\tau_{r}\right) L_{\tau_{r}}^{k}, L_{\tau}=L_{\tau_{r}}\right) \\
& \leq\left(2 a_{k}+3 c_{2}\right) \varepsilon R_{\tau} \times \frac{\sigma_{0}^{k} L_{\tau}^{k}}{(k+1)} \quad\left(\text { as } \sigma\left(\tau_{r}\right) \geq \sigma_{0}^{k},\|p-r\| \leq 2 R_{\tau}\right) \\
& \left.\leq \frac{\beta_{0}^{\prime}\left(2 a_{k}+3 c_{2}\right) \varepsilon}{(k+1)} \times \sigma_{0}^{k} L_{\tau}^{k+1} \quad \text { (as the edge-radius ratio is } \beta_{0}^{\prime}\right) \\
& <\sigma_{0}^{k+1} L_{\tau}^{k+1} .
\end{aligned}
$$

In our algorithm we pump all $j$-slivers for $j \in\{3, \ldots, k\}$ from $\operatorname{Del}_{T \mathbb{M}}^{\omega}(\mathcal{P}) \cup$ $\operatorname{Faces}^{\omega}(\mathcal{P})$. From Lemma 25, we can see that, if we remove also $(k+1)$-slivers from $\operatorname{Del}_{T \mathbb{M}}^{\omega}(\mathcal{P})$, then, if $\mathcal{P}$ is dense enough wrt the sliverity bound $\sigma_{0}$, specifically if

$$
\varepsilon<\frac{\sigma_{0}(k+1)}{\beta_{0}^{\prime}\left(3 c_{2}+2 a_{k}\right)}
$$

then there will be no $(k+1)$-slivers in $\operatorname{Del}_{T \mathbb{M}}^{\omega}(\mathcal{P})$. The proof of the above claim is the following. If a $(k+1)$-simplex $\tau$ remains in the final complex $\operatorname{Del}_{T \mathbb{M}}^{\omega}(\mathcal{P})$ returned by the algorithm, it cannot be a sliver nor its subfaces. But as the sample is dense (i.e $\left.\varepsilon<\frac{\sigma_{0}(k+1)}{\beta_{0}^{\prime}\left(3 c_{2}+2 a_{k}\right)}\right)$ and none of its subfaces are slivers, Lemma 25 implies that $\tau$ is a $(k+1)$-sliver. Hence we have reached a contradiction. So we will have no $(k+1)$-simplices in $\operatorname{Del}_{T \mathbb{M}}^{\omega}(\mathcal{P})$.

\section{Conclusion}

We have given the first algorithm that is able to reconstruct a smooth manifold in a time that depends only linearly on the dimension of the ambient space. We believe that our algorithm is of great interest when the dimension of the manifold is small, even if it is embedded in a space of very high dimension. This situation is quite common in practical applications in machine learning.

The algorithm is rather simple. The basic ingredients we need are data structures for constructing weighted Delaunay triangulations in $k$-flats. We intend to implement the algorithm in the near future, using an extension of the code developped for constructing Delaunay triangulations in any fixed dimension 5 .

In practice, the dimension of $\mathbb{M}$ is usually not known. We can use the algorithms given in [21, 15] to estimate the dimension $k$ of the manifold and the tangent space at each sample point. One interesting feature of our approach is that it is pretty robust and still works if we only have approximate tangent spaces at the sample points.

We forsee other applications of the tangential complex and of our construction each time computations in the tangent space of a manifold are required, e.g. for dimensionality reduction and approximating the Laplace Beltrami operator 4. 


\section{References}

[1] N. Amenta, S. Choi, T. K. Dey, , and N. Leekha. A simple algorithm for homeomorphic surface reconstruction. Intl. Journal of Computational Geometry and Application, 12:125-141, 2002.

[2] N. Amenta, S. Choi, T. K. Dey, and Leekha. A Simple Algorithm for Homeomorphic Surface Reconstruction. Internat. Journal of Comput. Geom. and Applications, 12(1-2):125-141, 2002.

[3] M. Belkin and P. Niyogi. Laplacian eigenmaps and spectral techniques for embedding and clustering. Advances in Neural Information Processing Systems, 2001.

[4] M. Belkin, J. Sun, and Y. Wang. Discrete laplace operator on meshed surfaces. In Proc. ACM Symp. on Computational Geometry, 2008.

[5] J-D. Boissonnat, O. Devillers, and S. Hornus. Incremental construction of the Delaunay triangulation and the Delaunay graph in medium dimension. ACM Symp. on Computational Geometry, 2009.

[6] J-D. Boissonnat and J. Flötotto. A coordinate system associated with points scattered on a surface. Journal of ACM, 36:161-174, 2004.

[7] J. D. Boissonnat, L. L. Guibas, and S. Y. Oudot. Manifold reconstruction in Arbitary Dimensions using Witness Complexes. In Proc. ACM Symp. on Computational Geometry, pages 193-204, 2007.

[8] J-D. Boissonnat and S. Oudot. Provably Good Sampling and Meshing of Surfaces. Graphical Models, 67:405-451, 2005.

[9] J-D. Boissonnat and M. Teillaud. Effective Computational Geometry for Curve and Surfaces. Springer, 2006.

[10] G. E. Bredon. Topology and Geometry. Graduate Text in Mathematics, Springer, 1994.

[11] F. Chazal and A. Lieutier. Smooth Manifold Reconstruction from Noisy and Non Uniform Approximation with Guarantees. Comp. Geom: Theory and Applications, 40:156-170, 2008.

[12] F. Chazal and S. Y. Oudot. Towards Persistence-Based Reconstruction in Euclidean Spaces. In Proc. ACM Symp. on Computational Geometry, pages 232-241, 2008.

[13] S-W Cheng, T. K. Dey, H. Edelsbrunner, M. A. Facello, and S-H Teng. Sliver Exudation. Journal of ACM, 47:883-904, 2000.

[14] S-W. Cheng, T. K. Dey, and E. A. Ramos. Manifold Reconstruction from Point Samples. In Proc. ACM-SIAM Symp. Discrete Algorithms, pages 1018-1027, 2005.

[15] S-W Cheng, Y. Wang, and Z Wu. Provable Dimension Detection using Principle Component Analysis. Intl. Journal of Computational Geometry and Application, 18 (5):415-440, 2008. 
[16] D. Cohen-Steiner and T. K. F. Da. A greedy Delaunay Based Surface Reconstruction Algorithm. The Visual Computer, 20:4-16, 2004.

[17] T. K. Dey. Curve and Surface Reconstruction: Algorithms with Mathematical Analysis. Cambridge University Press, 2006.

[18] D. L. Donoho and C. Grimes. Hessian eigenmaps: new locally linear embedding techniques for high dimensional data. Proceedings of the Natural Academy of Sciences, 100:5591-5596, 2003.

[19] J. Flötotto. A coordinate system associated to a point cloud issued from a manifold: definition, properties and applications. $\mathrm{PhD}$ thesis, Université of Nice Sophia-Antipolis, 2003.

[20] D Freedman. Efficient simplicial reconstructions of manifolds from their samples. IEEE Trans. on Pattern Analysis and Machine Intelligence, 24(10), 2002.

[21] J. Giesen and U. Wagner. Shape dimension and intrinsic metric from samples of manifolds. In Proc. ACM Symp. on Computational Geometry, pages 329-337, 2003.

[22] M. Gopi, S. Khrisnan, and C. T. Silva. Surface Reconstruction based on Lower Dimensional Localized Delaunay Triangulation. Eurographics, 19 (3), 2000

[23] A. Hatcher. Algebraic Topology. Cambridge University Press, New York, 2002.

[24] S. Lafon and A. B. Lee. Diffusion Maps and Coarse-Graining: A Unified Framework for Dimensionality Reduction, Graph Partitioning, and Data Set Parameterization. IEEE Transactions on Pattern Analysis and $\mathrm{Ma}$ chine Intelligence, 28:1393-1403, 2006.

[25] X-Y Li. Generating Well-Shaped $d$-dimensional Delaunay Meshes. Theoretical Computer Science, 296(1):145-165, 2003.

[26] S. Nadler, B.and Lafon, R. R. Coifman, and Kevrekidis. I. G. Diffusion Maps, Spectral Clustering, Eigenfunctions of Fokker-Planck Operators. Neural Information Processing Systems, 18, 2005.

[27] P. Niyogi, S. Smale, and S. Weinberger. Finding the Homology of Submanifolds with High Confidence from Random Samples. Discrete and Computational Geometry, 39(1-3), March 2008.

[28] S. T. Roweis and L. K. Saul. Nonlinear dimensionality reduction by locally linear embedding. Science, 290:2323-2326, 2000.

[29] H. Sebastian Seung and Daniel D. Lee. The manifold ways of perception. Science, 290:2268-2269, 2000.

[30] J. B. Tenenbaum, V. deSilva, and J. C. Langford. A global geometric framework for nonlinear dimensionality reduction. Science, 290:2319-2323, 2000 . 
[31] Z. Zhang and H. Zha. Principal manifolds and nonlinear dimension reduction via local tangent space alignment. SIAM Journal of Scientific Computing, 26(1):313-338, 2004.

\section{A Volume Lemma}

We want to bound the volume of $B(p, \epsilon \operatorname{lfs}(p)) \cap \mathbb{M}$. We assume that $\mathbb{M}$ be a smooth $k$-manifold embedded in $\mathbb{R}^{d}$. From [27], we have

Lemma 26 (Normal variation) Let $p, q$ be two points in $\mathbb{M}$ with $\|p-q\| \leq$ $\alpha \operatorname{lfs}(p), \alpha<\frac{1}{2}$, and $\theta=\angle N_{p} N_{q}$. We then have $2 \sin \frac{\theta}{2} \leq 1-\sqrt{1-4 \alpha}$, and the weaker bound $\sin \theta \leq 4 \alpha$.

Proof. It is proved in [27] (Propositions 6.2 and 6.3$)^{6}$ that $2 \sin \frac{\theta}{2} \leq 1-$ $\sqrt{1-\frac{4\|p-q\|}{\operatorname{lfs}(p)}} \leq 1-\sqrt{1-4 \alpha}$.

For all point $x \in \mathbb{M}$, we define the following projection map $f_{x}: B(x, \varepsilon \operatorname{lfs}(x)) \cap$ $\mathrm{M} \rightarrow T_{x}$

Lemma 27 For all points $p \in B(x, \varepsilon \operatorname{lfs}(x))$, the derivative $d f_{x}$ is nonsingular, where $\varepsilon<\frac{1}{2}$.

Proof. Let $d f_{x}$ be singular for some point $p \in B(x, \varepsilon \operatorname{lfs}(x)) \cap \mathbb{M}$. This implies that vector $\overrightarrow{p f_{x}(p)}$ is in $T_{p}$. But $\overrightarrow{p f_{x}(p)}$ is perpendicular to $T_{x}$, i.e, vector $\overrightarrow{p f_{x}(p)}$ is in $N_{p}$. Hence we have a contradiction from Lemma 26, as $\angle N_{p} N_{x} \leq \arcsin 2 \varepsilon<\pi / 2$.

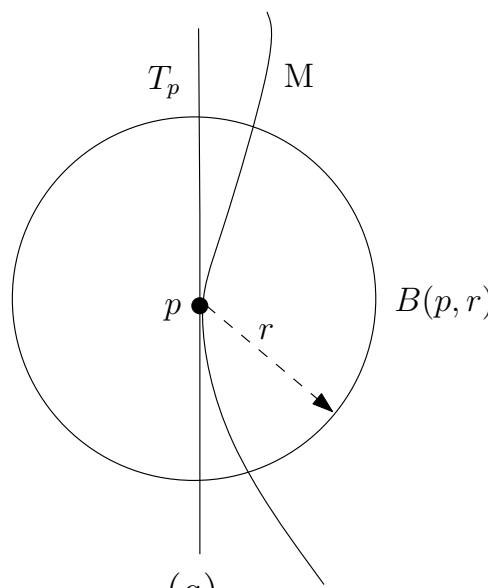

(a)

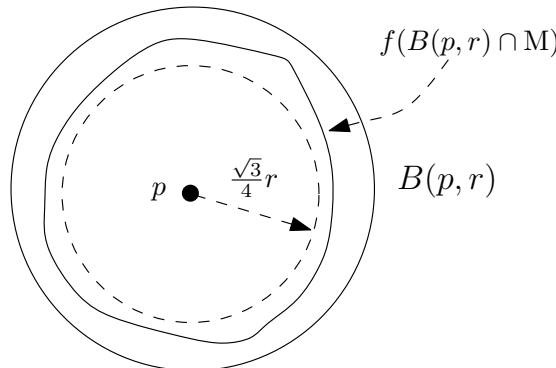

(b)

Figure 6: Refer to Lemma 28. In the figure $r=\varepsilon \operatorname{lfs}(p)$ and $f$ is the projection map of $\operatorname{vol}(B(p, r) \cap \mathbb{M})$ on $T_{p}$.

Lemma 28 Let $A=B(p, r) \cap \mathbb{M}$ where $r \leq \varepsilon \operatorname{lfs}(p)$. Then, $\phi_{k} r^{k} \geq \operatorname{vol}(A) \geq$ $\phi_{k} r^{k} \cos ^{k} \theta$, where $\phi_{k}$ is the volume of $k$-dimensional unit ball, $\sin \theta=\varepsilon / 2$ and $\varepsilon<\frac{1}{2}$.

\footnotetext{
${ }^{6}$ Although the tighter bound given here is (surprisingly) not explicitly stated in 27 .
} 
Proof. Let $B=B(p, r) \cap T_{p}$ be the $k$-dimensional ball radius $r \cos \theta$ centered at $p$ lying in $T_{p}$. Let $f_{p}^{A}=\left\{f_{p}(x) \mid \forall x \in A\right\}$. We will show that $B \subseteq f_{p}^{A}$. Since $f_{p}$ is a projection we have

$$
\operatorname{vol}(B) \geq \operatorname{vol}(A) \geq \operatorname{vol}\left(f_{p}^{A}\right) \geq \operatorname{vol}\left(B^{k}(p, r \cos \theta)\right) .
$$

To prove that $B \subseteq f_{p}^{A}$, notice that $f_{p}$ is an open map whose derivative is nonsingular for all $x \in A$, from Lemma 27. Therefore $f_{p}$ is locally invertable and there exists a ball $B_{s}^{k}=B(p, s) \cap T_{p}$ of radius $s$ s.t $f_{p}^{-1}\left(B_{s}^{k}\right) \subseteq A$. Let $s_{\text {sup }}=\sup \left\{s \mid f_{p}^{-1}\left(B_{s}^{k}\right) \subseteq A\right\}$. Then we have $f_{p}^{-1}\left(B_{\delta}^{k}\right) \subseteq A$, where $\delta<s_{\text {sup }}$. For all $x \in \operatorname{int}\left(B_{s_{\text {sup }}}^{k}\right)$, then $f_{p}^{-1}(x) \in A$. Let $q$ in the closure of $A$ such that either (i) $f_{p}$ is singular at $q$ or (ii) $q \notin A$. From Lemma 27 we know that case (i) is not possible, therefore $q \notin A$, then $\|p-q\|=r \leq \varepsilon \operatorname{lfs}(p)$. The angle between the line $p q$ and $p f_{p}(q)$ is less than $\theta$.

From, Lemma 28 we have

Corollary 1 Let $A=B(p, r) \cap \mathbb{M}$ where $r \leq \varepsilon \operatorname{lfs}(\mathbb{M})$. Then, $\phi_{k} r^{k} \geq \operatorname{vol}(A) \geq$ $\phi_{k} r^{k} \cos ^{k} \theta>\phi_{k} r^{k} / 2^{k}$, where $\phi_{k}$ is volume of $k$-dimensional unit ball, $\sin \theta=$ $\varepsilon / 2$ and $\varepsilon<\frac{1}{2}$.

\section{B Properties of simplices}

Lemma 29 (Volume of A SIMPLex) The volume of a $j$-simplex whose edges have length at most $L$ is at most $\frac{L^{j}}{j !}$.

Proof. $\operatorname{vol}(\tau)=\frac{1}{j !} \operatorname{det}\left|p_{1}-p_{0}, \ldots, p_{j}-p_{0}\right| \leq \frac{1}{j !}\left\|p_{1}-p_{0}\right\| \times \ldots \times\left\|p_{j}-p_{0}\right\| \leq \frac{L^{j}}{j !}$.

Lemma 30 (Height of a simplex) Let $\tau$ be a $j$-simplex. The distance $D_{p}$ between a vertex $p$ of $\tau$ and the affine hull of $\tau_{p}=\tau \backslash\{p\}$ is at least $\frac{j ! \rho(\tau)^{j-1} \sigma(\tau)}{2^{j-1}} L_{\tau}$.

Proof. Using the previous lemma and the definition of the sliver measure, we get

$$
D_{p}=\frac{j \operatorname{vol}(\tau)}{\operatorname{vol}\left(\tau_{p}\right)} \geq \frac{j \sigma(\tau) L_{\tau}^{j}}{\frac{\left(2 R_{\tau}\right)^{j-1}}{(j-1) !}} \geq \frac{j ! \rho(\tau)^{j-1} \sigma(\tau)}{2^{j-1}} L_{\tau} .
$$

from which the claim follows.

Lemma 31 Let $\tau$ be a $j$-dimensional simplex of $\operatorname{Del}^{\omega}(\mathcal{P})$ then

1. $\forall z \in \operatorname{aff}\left(\operatorname{Vor}^{\omega}(\tau)\right)$ and $\forall p, x \in \mathcal{P}$ we have $\|x-z\| \leq \frac{\|p-z\|}{\sqrt{1-4 \omega_{0}^{2}}}$.

2. $R_{\tau} \leq \frac{R_{\tau}^{\prime}}{\sqrt{1-4 \omega_{0}^{2}}}$.

3. $\forall z \in \operatorname{aff}\left(\operatorname{Vor}^{\omega}(\tau)\right)$ and $x \in \tau, Z=\sqrt{\|x-z\|^{2}-\omega^{2}(x)} \geq R_{\tau}^{\prime}$.

Proof. 
1. Observe that $o_{\tau}$ is the closest point to $x$ in $\operatorname{aff}\left(\operatorname{Vor}^{\omega}(\tau)\right)$. If $\|z-x\| \leq\|z-p\|$ then the lemma is proved. Hence assume that $\|z-x\|>\|z-p\|$. Since $z \in \operatorname{aff}\left(\operatorname{Vor}^{\omega}(\tau)\right)$

$$
\begin{aligned}
\|p-z\|^{2} & =\|x-z\|^{2}+\omega^{2}(p)-\omega^{2}(x) \\
& \geq\|x-z\|^{2}-\omega^{2}(x) \\
& \geq\|x-z\|^{2}-\omega_{0}^{2}\|p-x\|^{2} \\
& \geq\|x-z\|^{2}-\omega_{0}^{2}(\|p-z\|+\|z-x\|)^{2} \\
& >\|x-z\|^{2}-4 \omega_{0}^{2}\|x-z\|^{2} \\
& =\left(1-4 \omega_{0}^{2}\right)\|x-z\|^{2} .
\end{aligned}
$$

2. For all vertices $x$ of $\tau, l_{x} \leq 2 R_{\tau}$ and $\omega(x) \leq 2 \omega_{0} R_{\tau}$. From definition we have

$$
\begin{aligned}
R_{\tau}^{\prime} & \geq \min _{x \in \tau} \sqrt{\left\|c_{\tau}-x\right\|-\omega^{2}(x)} \\
& \geq \sqrt{1-4 \omega_{0}^{2}} R_{\tau} .
\end{aligned}
$$

3. We know that $o_{\tau}=\operatorname{aff}\left(\operatorname{Vor}^{\omega}(\tau)\right) \cap \operatorname{aff}(\tau)$. Therefore

$$
Z^{2}=\|x-z\|^{2}-\omega^{2}(x)=\left\|x-o_{\tau}\right\|^{2}+\left\|o_{\tau}-z\right\|^{2}-\omega^{2}(x)=R_{\tau}^{\prime 2}+\left\|o_{\tau}-z\right\|^{2} \geq R_{\tau}^{\prime 2} .
$$

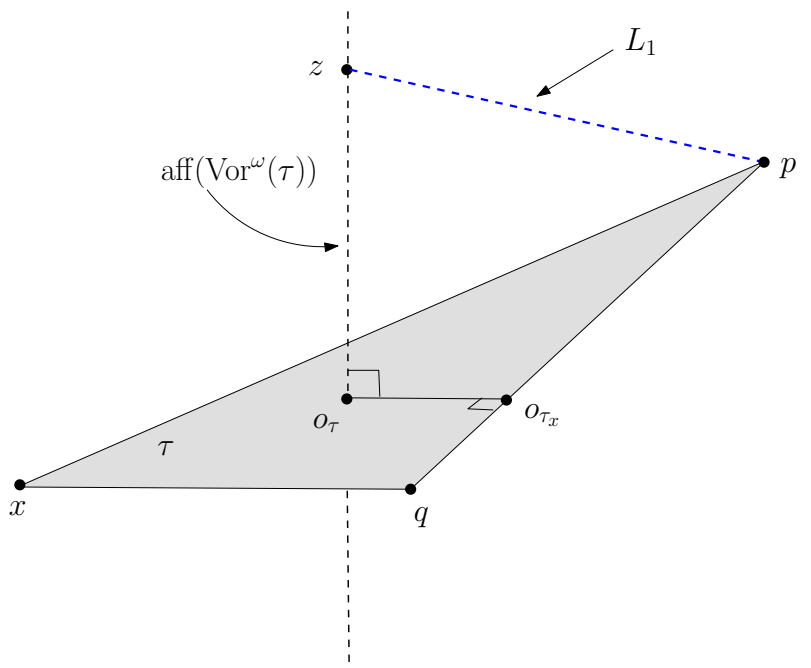

Figure 7: For the proofs of Lemmas 31 and 32 .

Lemma 32 Let $\tau$ be a $j$-dimensional simplex of $\operatorname{Del}^{\omega}(\mathcal{P})$ and $p \in \tau$ s.t.

1. There exists $z \in \operatorname{aff}\left(\operatorname{Vor}^{\omega}(\tau)\right.$ s.t. $\|z-p\| \leq L_{1}$.

2. $\|p-x\| \leq L_{2}$ for all vertices $x$ of $\tau$.

3. $R_{\tau}^{\prime} \leq \gamma_{0} L_{\tau}$. 
$H_{x}=\operatorname{dist}\left(o_{\tau}, \operatorname{aff}\left(\tau_{x}\right)\right) \leq L_{1}+\left(1+\gamma_{0}+\omega_{0}\right) L_{2}$ for all vertices $x$ of $\tau$.

Proof. Let $x \in \tau$ and $\tau_{x}=\tau \backslash\{x\} . H_{x}$ is equal to $\left\|o_{\tau}-o_{\tau_{x}}\right\|$. By Lemma 31 $\|x-z\| \leq \frac{L_{1}}{\sqrt{1-4 \omega_{0}^{2}}}$. Let $q$ be a vertex of $\tau_{x}$. We have

$$
\begin{aligned}
\left\|o_{\tau}-o_{\tau_{x}}\right\| & \leq\left\|o_{\tau}-p\right\|+\|p-q\|+\left\|q-o_{\tau_{x}}\right\| \\
& \leq\|z-p\|+\|p-q\|+\left\|q-o_{\tau_{x}}\right\| \\
& \leq L_{1}+L_{2}+\sqrt{R_{\tau_{x}}^{\prime 2}+\omega^{2}(q)} \\
& \leq L_{1}+L_{2}+\sqrt{\gamma_{0}^{2} L_{2}^{2}+\omega_{0}^{2} L_{2}^{2}} \\
& \leq L_{1}+\left(1+\gamma_{0}+\omega_{0}\right) L_{2}
\end{aligned}
$$

\section{Proofs}

Proof. (Lemma 6) Assume for a contradiction that there exist a point $x \in$ $\operatorname{Vor}^{\omega}(p) \cap T_{p}$ s.t $\|p-x\|>c_{1} \varepsilon \operatorname{lfs}(p)$. Let $q$ be a point on the segment $p x$ such that $\|p-q\|=c_{1} \varepsilon \operatorname{lfs}(p) / 2$. Let $q^{\prime}$ be the point nearest to $q$ on $\mathbb{M}$. From Lemma 2. we have $\left\|q-q^{\prime}\right\| \leq c_{1}^{2} \varepsilon^{2} \operatorname{lfs}(p) / 2$.

Hence, $\left\|p-q^{\prime}\right\| \leq\|p-q\|+\left\|q-q^{\prime}\right\|<\frac{c_{1}}{2} \varepsilon\left(1+c_{1} \varepsilon\right) \operatorname{lfs}(p)$. From the 1Lipschitz property, $\operatorname{lfs}\left(q^{\prime}\right) \leq \operatorname{lfs}(p)+\left\|p-q^{\prime}\right\|<\left(1+\frac{c_{1}}{2} \varepsilon\left(1+c_{1} \varepsilon\right)\right) \operatorname{lfs}(p)$, which yields

$\left\|p-q^{\prime}\right\| \geq\|p-q\|-\left\|q-q^{\prime}\right\|>\frac{c_{1}}{2} \varepsilon\left(1-c_{1} \varepsilon\right) \operatorname{lfs}(p)>\frac{c_{1}\left(1-c_{1} \varepsilon\right)}{2+c_{1} \varepsilon\left(1+c_{1} \varepsilon\right)} \varepsilon \operatorname{lfs}\left(q^{\prime}\right)>\varepsilon \operatorname{lfs}\left(q^{\prime}\right)$

if $c_{1}\left(1-c_{1} \varepsilon\right)>2+c_{1} \varepsilon\left(1+c_{1} \varepsilon\right)(*)$. Hence there exist a point $t \in \mathcal{P}$, s.t $\left\|q^{\prime}-t\right\| \leq \varepsilon \operatorname{lfs}\left(q^{\prime}\right)<\varepsilon\left(1+\frac{c_{1}}{2} \varepsilon\left(1+c_{1} \varepsilon\right)\right) \operatorname{lfs}(p)$. We thus have (using again $\left(^{*}\right)$ )

$$
\|q-t\| \leq\left\|q-q^{\prime}\right\|+\left\|q^{\prime}-t\right\|<\frac{c_{1}}{2} \varepsilon \operatorname{lfs}(p) .
$$

From Fig. 8, we can see that $\angle p t x>\pi / 2$. This implies that $\|p-x\|^{2}-\| x-$ $t\left\|^{2}-\right\| p-t \|^{2}>0$. So,

$$
\begin{aligned}
\|x-p\|^{2}-\|x-t\|^{2}-\omega^{2}(p)+\omega^{2}(t) & \geq\|x-p\|^{2}-\|x-t\|^{2}-\omega^{2}(p) \\
& \geq\|x-p\|^{2}-\|x-t\|^{2}-\omega_{0}^{2} \cdot\|p-t\|^{2} \\
& \geq\|x-p\|^{2}-\|x-t\|^{2}-\|p-t\|^{2} \quad\left(\text { as } \omega_{0} \in[0,1 / 2)\right) \\
& >0 .
\end{aligned}
$$

This implies $x \notin \operatorname{Vor}^{\omega}(p)$, which contradicts our initial assumption. We conclude that $\operatorname{Vor}^{\omega}(p) \cap T_{p} \subseteq B\left(p, c_{1} \varepsilon \operatorname{lfs}(p)\right)$.

Inequality $(*)$ is satisfied for $\varepsilon<\frac{1}{2 c_{1}}\left(-c_{1}-1+\sqrt{c_{1}^{2}+6 c_{1}-7}\right)$. The quantity on the right hand side is positive when $c_{1}>2$. We maximize the bound on $\varepsilon$ by taking $c_{1}=3+\sqrt{2} \approx 4.41$. Inequality $(*)$ then holds for $\varepsilon<0.09$.

Proof. (Lemma 7) 1. Since $p q \in \operatorname{Del}_{p}^{\omega}(\mathcal{P})$ therefore $T_{p} \cap \operatorname{Vor}^{\omega}(p q) \neq \emptyset$. Let $x \in T_{p} \cap \operatorname{Vor}^{\omega}(p q)$. From Lemma 6 we have $\|p-x\| \leq c_{1} \varepsilon l f s(p)$. If $\|q-x\| \leq$ 


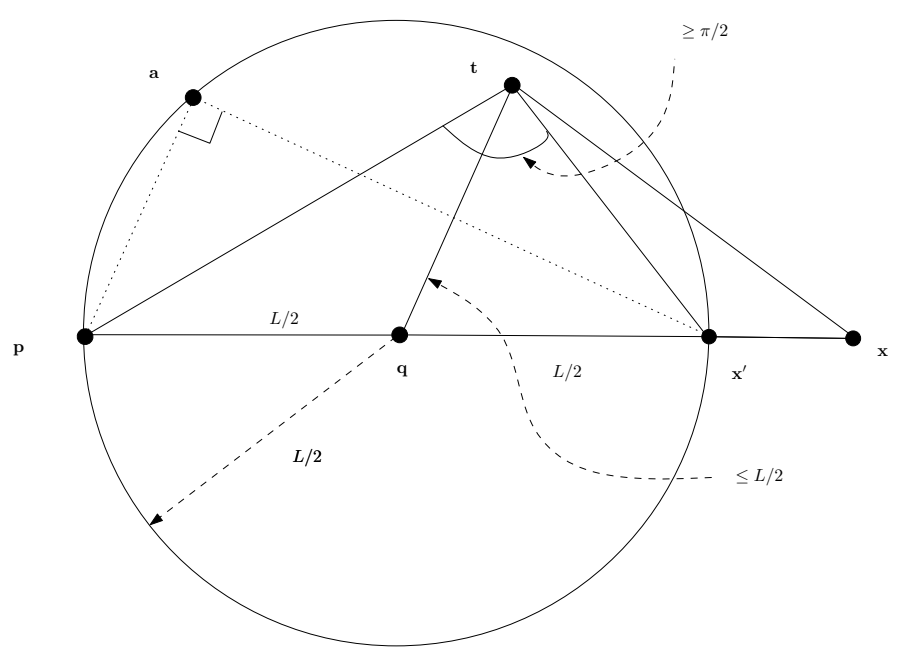

Figure 8: Refer to Lemma 6. $x^{\prime}$ is a point on the line segment such that $\left\|p-x^{\prime}\right\|=c_{1} \varepsilon \operatorname{lfs}(p), L=c_{1} \varepsilon \operatorname{lfs}(p), \angle p a x^{\prime}=\pi / 2$ and $\angle p t x \geq \angle p t x^{\prime}>\pi / 2$.

$\|p-x\|$ then the inequality holds. Otherwise, $\|p-q\| \leq\|p-x\|+\|q-x\|<$ $2\|q-x\|$. Since $x \in T_{p} \cap \operatorname{Vor}^{\omega}(\{p, q\})$, we have from Lemma 31 (Appendix B), $\|q-x\| \leq \frac{\|p-x\|}{\sqrt{1-4 \omega_{0}^{2}}}$.

Hence we get $\|p-q\| \leq\|p-x\|+\|q-x\| \leq c_{1}\left(1+1 / \sqrt{1-4 \omega_{0}^{2}}\right) \varepsilon \operatorname{lfs}(p)=$ $c_{2} \varepsilon \operatorname{lfs}(p)$.

2. From the definition of $\operatorname{Del}_{T \mathbb{M}}^{\omega}(\mathcal{P})$, there exist a vertex $r$ in $\tau$ such that $[p q] \in \operatorname{Del}_{r}^{\omega}(\mathcal{P})$. From 1, $\|r-p\|$ and $\|r-q\|$ are at most $c_{2} \varepsilon \operatorname{lfs}(r)$. From the 1-Lipschitz property of lfs and $3 c_{2} \varepsilon<1$, we have lfs $(r) \leq \operatorname{lfs}(p)+\|p-r\| \leq$ $\frac{\operatorname{lfs}(p)}{1-c_{2} \varepsilon} \leq \frac{3}{2} \operatorname{lfs}(p)$. We conclude that $\|p-q\| \leq\|p-r\|+\|r-q\| \leq 3 c_{2} \varepsilon \operatorname{lfs}(p)$.

3. Let $z \in \operatorname{Vor}^{\omega}(\tau) \cap T_{p}$, and $Z=\sqrt{\|z-p\|^{2}-\omega^{2}(p)}$. By definition the ball centered at $z$ with radius $Z$ is orthogonal to the weighted vertices of $\tau$. From Lemma 31 (Appendix B), we have $Z \geq R_{\tau}^{\prime}$. Hence it suffices to prove $Z \leq \beta_{0} L_{\tau}$. Since $z \in \operatorname{Vor}^{\omega}(\tau) \cap T_{p}$, we deduce from Lemma 6 that $\|z-p\| \leq c_{1} \varepsilon \operatorname{lfs}(p)$. Therefore

$$
Z=\sqrt{\|z-p\|^{2}-\omega^{2}(p)} \leq\|z-p\| \leq c_{1} \varepsilon \operatorname{lfs}(p) \leq c_{1}\left(\frac{\varepsilon}{\delta}\right) \delta \operatorname{lfs}(p) .
$$

For any vertex $x$ of $\tau$, we have $\|p-x\| \leq c_{2} \varepsilon \mathrm{lfs}(p)$ (By 1.). Since $2 c_{2} \varepsilon \leq 1$ and lfs is 1-Lipschitz, $\operatorname{lfs}(p) \leq 3 \operatorname{lfs}(x) / 2$. Therefore, taking for $x$ a vertex of the shortest edge of $\tau$, we have

$$
Z \leq c_{1}\left(\frac{\varepsilon}{\delta}\right) \delta \operatorname{lfs}(p) \leq c_{1}\left(\frac{\varepsilon}{\delta}\right) \delta \times \frac{3 \operatorname{lfs}(x)}{2} \leq\left(\frac{3 c_{1} \varepsilon}{2 \delta}\right) L_{\tau}=\beta_{0} L_{\tau} .
$$

From Lemma 31 we have $R_{\tau} \leq \frac{R_{\tau}^{\prime}}{\sqrt{1-4 \omega_{0}^{2}}}$. There fore radius-edge ratio of $\tau$ is $\beta_{0}^{\prime}=\frac{\beta_{0}}{\sqrt{1-4 \omega_{0}^{2}}}$.

Proof. (Lemma 9) Let $\phi=\left[p_{1}, \ldots, p_{k+2}\right]$ be an inconsistent configuration witnessed by $p_{i}, p_{j}, p_{l}$. 
1. If $p q$ is an edge of $\phi$, Lemma 8 implies that $\left\|p_{i}-c_{\phi}\right\| \leq 2 c_{4} \varepsilon \mathrm{lfs}\left(p_{i}\right)$ and that $\left\|x-c_{\phi}\right\| \leq c_{5} \varepsilon \mathrm{lfs}(x), \forall x \in \phi$. Let $p, q$ be two vertices of $\phi$, then

$$
\begin{aligned}
\|p-q\| & \leq\left\|p-c_{\phi}\right\|+\left\|q-c_{\phi}\right\| \\
& \leq 2 c_{5} \varepsilon \operatorname{lfs}\left(p_{i}\right) \\
& \leq 4 c_{5} \varepsilon \operatorname{lfs}(p)
\end{aligned}
$$

the last inequality follows from the fact that lfs is 1-Lipschitz function and $2 c_{5} \varepsilon<1$.

2. Let $Z=\sqrt{\left\|c_{\phi}-p_{i}\right\|^{2}-\omega^{2}\left(p_{i}\right)}$. Since $c_{\phi} \in \operatorname{Vor}^{\omega}(\tau)$, the ball centered at $c_{\phi}$ with radius $Z$ is orthogonal to weighted vertices of $\tau$. From Lemma 31, we have $Z \geq R_{\tau}^{\prime}$. Hence if we can show that $Z \leq \beta_{1} L_{\tau}$ then we are done. Using $\left\|p_{i}-c_{\phi}\right\| \leq 2 c_{4} \varepsilon \mathrm{lfs}\left(p_{i}\right)$ we get

$$
Z=\sqrt{\left\|c_{\phi}-p_{i}\right\|-\omega^{2}\left(p_{i}\right)} \leq\left\|c_{\phi}-p_{1}\right\| \leq 2 c_{4} \varepsilon \operatorname{lfs}\left(p_{i}\right) .
$$

For all vertices $x$ in $\tau$ we have from Lemma $9(1)\left\|p_{i}-x\right\| \leq 4 c_{5} \varepsilon \operatorname{lfs}\left(p_{i}\right)$. Since $16 c_{5} \varepsilon \leq 1$ we get from the 1 -Lipschitz property of lfs, $\operatorname{lfs}\left(p_{i}\right) \leq 5 \operatorname{lfs}(x) / 4$. Therefore

$Z \leq 2 c_{4}\left(\frac{\varepsilon}{\delta}\right) \delta \operatorname{lfs}\left(p_{i}\right) \leq 2 c_{4}\left(\frac{\varepsilon}{\delta}\right) \delta \times \frac{5 \operatorname{lfs}(x)}{4} \leq \frac{5 c_{4} \varepsilon \operatorname{lfs}(x)}{2} \leq\left(\frac{5 c_{4} \varepsilon}{2 \delta}\right) L_{\tau}=\beta_{1} L_{\tau}$.

From Lemma 31, we have $R_{\tau} \leq \frac{R_{\tau}^{\prime}}{\sqrt{1-4 \omega_{0}^{2}}}$. Therefore the radius-edge ratio for $\tau$ is $\beta_{1}^{\prime}=\frac{\beta_{1}}{\sqrt{1-4 \omega_{0}^{2}}}$.

Proof. (Lemma 10) Let $\tau$ be a simplex of $\operatorname{Del}_{T \mathbb{M}}^{\omega}(\mathcal{P})$. Then there exists a vertex $p$ s.t $p \in \tau_{1}, \tau \subseteq \tau_{1}$ and $\tau_{1}$ belongs to $\operatorname{Del}_{p}^{\omega}(\mathcal{P})$. From Lemma 6 we have $\left\|p-c_{p}\right\| \leq c_{1} \varepsilon \operatorname{lfs}(p) \leq 2 c_{1} \varepsilon \operatorname{lfs}(x)$ where $c_{p}=\operatorname{Vor}^{\omega}\left(\tau_{1}\right) \cap$ for all vertices $x$ of $\tau$. The last inequality follows from the facts that lfs is 1-Lipschitz, $\|p-x\| \leq$ $3 c_{2} \varepsilon \operatorname{lfs}(p)$ (refer to Lemma 7) and $3 c_{2} \varepsilon<16 c_{2} \varepsilon<1$. We have from Lemma 31 $\left\|x-c_{p}\right\| \leq \frac{2 c_{1} \varepsilon l \mathrm{fs}(x)}{\sqrt{1-4 \omega_{0}^{2}}}$ as $c_{p} \in \operatorname{Vor}^{\omega}(\tau) \subseteq \operatorname{aff}\left(\operatorname{Vor}^{\omega}(\tau)\right)$. Therefore using Lemma 32 we get

$$
\begin{aligned}
\operatorname{dist}\left(o_{\tau}, \tau_{x}\right) & \leq \frac{2 c_{1} \varepsilon \operatorname{lfs}(x)}{\sqrt{1-4 \omega_{0}^{2}}}+3\left(1+\beta_{0}+\omega_{0}\right) c_{2} \varepsilon \operatorname{lfs}(x) \\
& \leq c_{6} \varepsilon \operatorname{lfs}(x)
\end{aligned}
$$

as $L_{1}, L_{2}, \gamma_{0}$ in Lemma 32 (Appendix B corresponds to $\frac{2 c_{1} \varepsilon \mathrm{lfs}(x)}{\sqrt{1-4 \omega_{0}^{2}}}, 3 c_{2} \varepsilon \mathrm{lfs}(x)$ (from Lemma 7 (1)) and $\beta_{0}$ (Lemma 7 (2))respectively. The proof is similar when $\tau$ belongs to $\operatorname{Faces}^{\omega}(\mathcal{P})$.

Proof. (Lemma 11) Assume w.l.o.g. that $\phi$ is witnessed by $p_{1}, p_{2}$ and $p_{k+2}$. Let $\tau=\phi \backslash p_{k+2}$ and $p_{i}$ be any vertex of $\tau$ (i.e. $i \in[1, k+1]$ ). Write, as usual, $\phi_{p_{i}}$ for the $k$-simplex $\phi \backslash\left\{p_{i}\right\}$. Let $o_{\phi_{i}}$ and $o_{\tau}$ be the orthocenters of $\phi_{p_{i}}$ and $\tau$ respectively. By Lemma 8 , we have that $\operatorname{dist}\left(c_{\phi}\right.$, aff $\left.(\tau)\right)=\left\|o_{\tau}-c_{\phi}\right\| \leq$ $2 c_{4} \varepsilon^{2} \operatorname{lfs}\left(p_{1}\right)$. This proves the lemma for $\tau$ (one of the $k$-subfaces of $\phi$ ).

To complete the proof, it is enough to consider the case of $\phi_{i}$. We have $\left\|o_{\tau}-p_{1}\right\| \leq\left\|c_{p_{1}}-p_{1}\right\| \leq c_{1} \varepsilon \operatorname{lfs}\left(p_{1}\right)$ and $\left\|p_{1}-p_{i}\right\| \leq c_{2} \varepsilon \operatorname{lfs}\left(p_{1}\right)$ (from Lemmas 6 
and 7 respectively). If $q$ denotes a common vertex of $\phi_{p_{i}}$ and $\tau$, we get, using Lemma 5 ,

$$
\begin{aligned}
\operatorname{dist}\left(\operatorname{aff}\left(\phi_{p_{i}}\right), o_{\tau}\right) & \leq\left\|o_{\tau}-q\right\| \sin \angle\left(\operatorname{aff}(\tau), \operatorname{aff}\left(\phi_{p_{i}}\right)\right. \\
& \leq\left\|o_{\tau}-q\right\|\left(\sin \angle\left(\operatorname{aff}(\tau), T_{q}\right)+\sin \angle\left(\phi_{p_{i}}, T_{q}\right)\right) \\
& \leq\left(\left\|o_{\tau}-p_{1}\right\|+\left\|p_{1}-q\right\|\right) 2 a_{k} \varepsilon \\
& \leq 2\left(c_{1}+c_{2}\right) a_{k} \varepsilon^{2} \operatorname{lfs}\left(p_{1}\right) .
\end{aligned}
$$

Since dist $\left(c_{\phi}, \operatorname{aff}\left(\phi_{p_{i}}\right)=\left\|o_{\phi_{i}}-c_{\phi}\right\| \leq \operatorname{dist}\left(\operatorname{aff}\left(\phi_{p_{i}}\right), o_{\tau}\right)+\left\|o_{\tau}-c_{\phi}\right\| \leq 2\left(c_{1}+c_{2}+\right.\right.$ $\left.c_{4}\right) a_{k} \varepsilon^{2} \operatorname{lfs}\left(p_{1}\right)$.

To conclude, we observe that, for any vertex $v$ of $\phi, \operatorname{lfs}\left(p_{1}\right) \leq \operatorname{lfs}(v)+\left\|p_{1}-v\right\|$ (Lemma 9), and use $\left\|p_{1}-v\right\| \leq 4 c_{5} \operatorname{lfs}\left(p_{1}\right)$ and $8 c_{5} \varepsilon \leq 1$.

Proof. (Lemma 12p Let $p$ be a vertex of $\phi_{p_{i}}$. By Lemma 9(2) and Lemma 5 we have $\sin \angle\left(\operatorname{aff}(\tau), T_{p}\right) \leq a_{k} \varepsilon$, and, by Lemma 2(2), we have $\sin \angle\left(p p_{i}, T_{p}\right) \leq$ $2 c_{5} \varepsilon$. We therefore have, $\sin \angle\left(p p_{i}, \operatorname{aff}(\tau)\right) \leq \sin \angle\left(p p_{i}, T_{p}\right)+\sin \angle\left(T_{p}, \operatorname{aff}(\tau)\right) \leq$ $\left(2 c_{5}+a_{k}\right) \varepsilon$. Hence

$$
D_{p_{i}}=\left\|p-p_{i}\right\| \sin \angle\left(p p_{i}, \operatorname{aff}(\tau)\right)<4\left(2 c_{5}+a_{k}\right) c_{5} \varepsilon^{2} \operatorname{lfs}\left(p_{i}\right),
$$

since $\left\|p-p_{i}\right\| \leq 4 c_{5} \varepsilon \mathrm{lfs}\left(p_{i}\right)$ from Lemma 9

Proof. (Lemma 13) For convenience, write $\nu=16 c_{5} c_{0} \varepsilon \leq 1 / 2$ and observe that $L N_{p} \subset B_{p}^{\prime \prime}=B(p, \nu \operatorname{lfs}(p))$ since, by Lemma $2, l_{p} \leq \frac{2 \varepsilon}{1-\varepsilon} \operatorname{lfs}(p) \leq 4 \varepsilon \operatorname{lfs}(p)$. We will count the number of points in $B_{p}^{\prime \prime} \cap \mathcal{P}$. Let $x$ and $y$ be two points of $B_{p}^{\prime \prime} \cap \mathcal{P}$. We have $\operatorname{lfs}(x), \operatorname{lfs}(y) \geq \operatorname{lfs}(p)(1-\nu) \geq \operatorname{lfs}(p) / 2$, since lfs is 1-Lipschitz. The balls $B\left(x, l_{x} / 2\right)$ and $B\left(y, l_{y} / 2\right)$ are disjoint, and, since $l_{x} \geq \delta \operatorname{lfs}(x) \geq \frac{\delta}{2} \operatorname{lfs}(p)$ (and similarly for $l_{y}$ ), the balls $B_{x}=B\left(x, \frac{\delta}{4} \operatorname{lfs}(p)\right)$ and $B_{y}=B\left(y, \frac{\delta}{4} \operatorname{lfs}(p)\right)$ are also disjoint. Observe that both balls $B_{x}$ and $B_{y}$ are contained in $B_{p}^{+}=$ $B(p, \mu \varepsilon \operatorname{lfs}(p))$ where $\mu=\frac{\nu}{\varepsilon}+\frac{\delta}{4 \varepsilon} \leq 16 c_{5} c_{0}+\frac{1}{4}$.

A packing argument now allows to conclude. Specifically, by Corollary 1 (see Appendix A), we have that $\operatorname{vol}\left(B_{x} \cap \mathbb{M}\right)>\phi_{k}\left(\frac{\delta \operatorname{lfs}(p)}{8}\right)^{k}$ and $\operatorname{vol}\left(B_{p}^{+} \cap\right.$ $\mathbb{M})<\phi_{k}(\mu \varepsilon \operatorname{lfs}(p))^{k}$, where $\phi_{k}$ is the volume of the $k$-dimensional unit ball. We conclude that the number of points of $\mathcal{P} \cap B_{p}^{\prime \prime}$ is less than $\left(\frac{8 \mu \varepsilon}{\delta}\right)^{k} \leq\left(128 c_{5} c_{0}+\right.$ $2)^{k} c_{0}^{k}=2^{O(k)}$. 


\section{Contents}

$\begin{array}{lll}1 & \text { Introduction } & 3\end{array}$

2 Definitions and preliminaries 5

2.1 Weighted Delaunay triangulation . . . . . . . . . . . . . . . 5

2.2 Sampling conditions $\ldots \ldots \ldots \ldots$. . . . . . . . . . . . . . . . . . . . . . . 6

$2.3 \quad$ Slivers and good simplices . . . . . . . . . . . . . . . . . . . . 7

2.4 Tangential Delaunay complex and inconsistent configurations . . 9

$\begin{array}{lll}3 & \text { Structural results } & 11\end{array}$

$3.1 \quad$ Properties of the simplices of the tangential Delaunay complex . 11

3.2 Properties of inconsistent configurations . . . . . . . . . . . 11

3.3 Range of weights for slivers and inconsistencies . . . . . . . . . . 13

$\begin{array}{lll}4 & \text { Inconsistencies removal } & \mathbf{1 4}\end{array}$

4.1 Algorithm: removing slivers and inconsistencies . . . . . . . . . . 14

4.2 Time complexity . . . . . . . . . . . . . . . . . . . . . . 18

4.3 Space complexity . . . . . . . . . . . . . . . . . . . . . 19

5 Topological and geometric guarantees 20

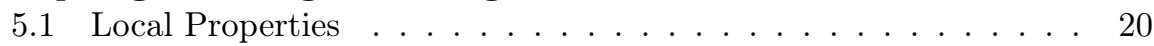

5.2 Isotopy . . . . . . . . . . . . . . . . . . . . . . . . 21

5.3 Hausdorff Distance . . . . . . . . . . . . . . . . . . . . . . . 24

\begin{tabular}{|ll|}
6 & Summary of results \\
\hline
\end{tabular}

\begin{tabular}{lll}
\hline $\mathbf{7}$ & Extensions & $\mathbf{2 6}$
\end{tabular}

$7.1 \quad$ Dimension and tangent space estimation. . . . . . . . . . . . . . 26

7.2 $\quad$ Removing the general position assumption . . . . . . . . . . . . 26

$\begin{array}{lll}8 & \text { Conclusion } & 27\end{array}$

\begin{tabular}{|ll}
\hline A Volume Lemma & 30
\end{tabular}

\begin{tabular}{|lr}
\hline B Properties of simplices & 31
\end{tabular}

\begin{tabular}{ll|}
\hline C Proofs & 33 \\
\hline
\end{tabular} 
Centre de recherche INRIA Sophia Antipolis - Méditerranée 2004, route des Lucioles - BP 93 - 06902 Sophia Antipolis Cedex (France)

Centre de recherche INRIA Bordeaux - Sud Ouest : Domaine Universitaire - 351, cours de la Libération - 33405 Talence Cedex Centre de recherche INRIA Grenoble - Rhône-Alpes : 655, avenue de l'Europe - 38334 Montbonnot Saint-Ismier

Centre de recherche INRIA Lille - Nord Europe : Parc Scientifique de la Haute Borne - 40, avenue Halley - 59650 Villeneuve d'Ascq Centre de recherche INRIA Nancy - Grand Est : LORIA, Technopôle de Nancy-Brabois - Campus scientifique 615, rue du Jardin Botanique - BP 101 - 54602 Villers-lès-Nancy Cedex

Centre de recherche INRIA Paris - Rocquencourt : Domaine de Voluceau - Rocquencourt - BP 105 - 78153 Le Chesnay Cedex

Centre de recherche INRIA Rennes - Bretagne Atlantique : IRISA, Campus universitaire de Beaulieu - 35042 Rennes Cedex Centre de recherche INRIA Saclay - Île-de-France : Parc Orsay Université - ZAC des Vignes : 4, rue Jacques Monod - 91893 Orsay Cedex 